\title{
Meanders as a scaling motif for understanding of floodplain soil microbiome and biogeochemical potential at the watershed scale
}

Paula B. Matheus Carnevali', Adi Lavy', Alex D. Thomas², Alexander Crits-Christoph ${ }^{3}$, Spencer Diamond ${ }^{1}$, Raphaël Méheust ${ }^{1,4}$, Matthew R. Olm³ ${ }^{3,5}$, Allison Sharrar ${ }^{1}$, Shufei Lei ${ }^{1}$, Wenming Dong ${ }^{6}$, Nicola Falco ${ }^{6}$, Nicholas Bouskill ${ }^{6}$, Michelle E. Newcomer ${ }^{6}$, Peter Nico ${ }^{6}$, Haruko Wainwright ${ }^{6}$, Dipankar Dwivedi ${ }^{6}$, Kenneth H. Williams ${ }^{6}$, Susan Hubbard ${ }^{6}$ and Jillian F. Banfield ${ }^{1,2,3,4,6,7^{*}}$ (1)

\begin{abstract}
Background: Biogeochemical exports from watersheds are modulated by the activity of microorganisms that function over micron scales. Here, we tested the hypothesis that meander-bound regions share a core microbiome and exhibit patterns of metabolic potential that broadly predict biogeochemical processes in floodplain soils along a river corridor.
\end{abstract}

Results: We intensively sampled the microbiomes of floodplain soils located in the upper, middle, and lower reaches of the East River, Colorado. Despite the very high microbial diversity and complexity of the soils, we reconstructed 248 quality draft genomes representative of subspecies. Approximately one third of these bacterial subspecies was detected across all three locations at similar abundance levels, and $\sim 15 \%$ of species were detected in two consecutive years. Within the meander-bound floodplains, we did not detect systematic patterns of gene abundance based on sampling position relative to the river. However, across meanders, we identified a core floodplain microbiome that is enriched in capacities for aerobic respiration, aerobic CO oxidation, and thiosulfate oxidation with the formation of elemental sulfur. Given this, we conducted a transcriptomic analysis of the middle floodplain. In contrast to predictions made based on the prominence of gene inventories, the most highly transcribed genes were relatively rare amoCAB and $n \times r A B$ (for nitrification) genes, followed by genes involved in methanol and formate oxidation, and nitrogen and $\mathrm{CO}_{2}$ fixation. Within all three meanders, low soil organic carbon correlated with high activity of genes involved in methanol, formate, sulfide, hydrogen, and ammonia oxidation, nitrite oxidoreduction, and nitrate and nitrite reduction. Overall, the results emphasize the importance of sulfur, one-carbon and nitrogen compound metabolism in soils of the riparian corridor.

(Continued on next page)

\footnotetext{
* Correspondence: jbanfield@berkeley.edu

'Department of Earth and Planetary Science, University of California, Berkeley, USA

${ }^{2}$ Department of Environmental Science, Policy, and Management, University of California, Berkeley, CA, USA

Full list of author information is available at the end of the article
}

(c) The Author(s). 2021 Open Access This article is licensed under a Creative Commons Attribution 4.0 International License, which permits use, sharing, adaptation, distribution and reproduction in any medium or format, as long as you give appropriate credit to the original author(s) and the source, provide a link to the Creative Commons licence, and indicate if changes were made. The images or other third party material in this article are included in the article's Creative Commons licence, unless indicated otherwise in a credit line to the material. If material is not included in the article's Creative Commons licence and your intended use is not permitted by statutory regulation or exceeds the permitted use, you will need to obtain permission directly from the copyright holder. To view a copy of this licence, visit http://creativecommons.org/licenses/by/4.0/ The Creative Commons Public Domain Dedication waiver (http://creativecommons.org/publicdomain/zero/1.0/) applies to the data made available in this article, unless otherwise stated in a credit line to the data. 


\begin{abstract}
(Continued from previous page)
Conclusions: The disparity between the scale of a microbial cell and the scale of a watershed currently limits the development of genomically informed predictive models describing watershed biogeochemical function. Meanderbound floodplains appear to serve as scaling motifs that predict aggregate capacities for biogeochemical transformations, providing a foundation for incorporating riparian soil microbiomes in watershed models. Widely represented genetic capacities did not predict in situ activity at one time point, but rather they define a reservoir of biogeochemical potential available as conditions change.
\end{abstract}

Keywords: Microbiome, Floodplain, Soil, Watershed, Genome-resolved metagenomics, Metatranscriptomics

\section{Background}

Watersheds are geographic areas that capture precipitation that is ultimately discharged into rivers and other larger water bodies. Of particular interest are watersheds in mountainous regions, as these are major sources of freshwater $[1,2]$. Within mountainous watersheds, complex interactions among vegetation, hydrology, geochemistry, and geology occur within and across watershed compartments, including across bedrock-soil-vegetation compartments of terrestrial hillslopes, across terrestrialaquatic interfaces, and within the fluvial system itself. Interactions within a reactive watershed typically vary as a function of disturbance as well as landscape position and topography. For example, interactions in an alpine region of a mountainous watershed are likely to be quite different from a lower montane floodplain region [3]. Floodplains, which extend from the river banks to the base of hillslopes, comprise the riparian zone (a vegetated interface between the river channel and the rest of the ecosystem), and are notable as they integrate inputs from all watershed compartments. They also display depositional gradients and features associated with past and current river channel positions. Unlike hillslopes, floodplains receive water and constituents either by surface runoff or groundwater discharge. They are typically significantly impacted by changes in river conditions and can be inundated when river flow and stage increase following snowmelt. Consequently, floodplains are dynamic compartments in which hydrobiogeochemical processes vary seasonally and potentially spatially. Overall, floodplains are important watershed regions in which microbial activity can modulate the form and abundance of nutrients and contaminants derived from hillslopes and river water prior to their export from the watershed.

Here, we conducted a study of floodplain soils of the mountainous East River ( $\mathrm{CO}$ ) watershed to investigate how patterns in the distribution of soil microorganisms and their associated functions and activities could contribute to geochemical gradients that impact riverine nutrient fluxes. We tested a "system of systems" approach [4] wherein meander-bound regions were selected as scaling motifs (repeating patterns along the river that can be used for ecosystem modeling at the watershed scale), in which microbially mediated biogeochemical processes that are shaped by reactions occurring at the micron scale might be representative of processes throughout the floodplain. Detailed analyses of meander-bound floodplain soils may reveal patterns that approximate watershed processes at the tens of kilometer scale, and could provide much needed input for watershed hydrobiogeochemical models. This study applied genome-resolved metagenomic and metatranscriptomic bioinformatics methods to large nucleic acid sequence datasets to investigate microbial community composition and distribution and to infer capacities for microbially mediated $\mathrm{C}, \mathrm{S}, \mathrm{H}$, and $\mathrm{N}$ cycling and in situ activity in floodplain soil microbial communities.

\section{Results}

\section{Metagenome overview}

Three meander-bound floodplains following the meandering pattern of the East River (Fig. 1 a ) were chosen for this study: one upstream (meander-bound floodplain G (Floodplain G) (Fig. 1 b)), one midstream (meander-bound floodplain L (Floodplain L) (Fig. $1 \mathrm{c})$ ), and one downstream (meander-bound floodplain $\mathrm{Z}$ (Floodplain Z) (Fig. 1 d)). Sample number was prioritized over sequencing depth to better resolve the types and distribution patterns of the most abundant organisms across the meander-bound floodplains ("floodplains" subsequently). An average 6.4 giga base pairs (Gbp; 3.2-11.5 Gbp) of sequencing data was obtained from 90 DNA extractions out of 94 floodplain soil samples collected in 2015. An average 12 Gbp (6.0-15.0 $\mathrm{Gbp}$ ) of sequencing data was obtained from the other four samples. In total, $\sim 0.6 \mathrm{Tbp}$ of DNA sequence information was acquired from samples collected in 2015 . Our strategy aimed to capture the most abundant members of the microbial community (instead of the whole community), so it is not surprising that an average 13\% (3-30\%) of reads mapped to their respective assemblies (Table S1, Additional file 1). This result also reflects the large (but variable) tail on the abundance distribution of microorganisms in soil [5]; and the communities captured by the smaller assemblies resulting from samples with lower sequencing depth. 


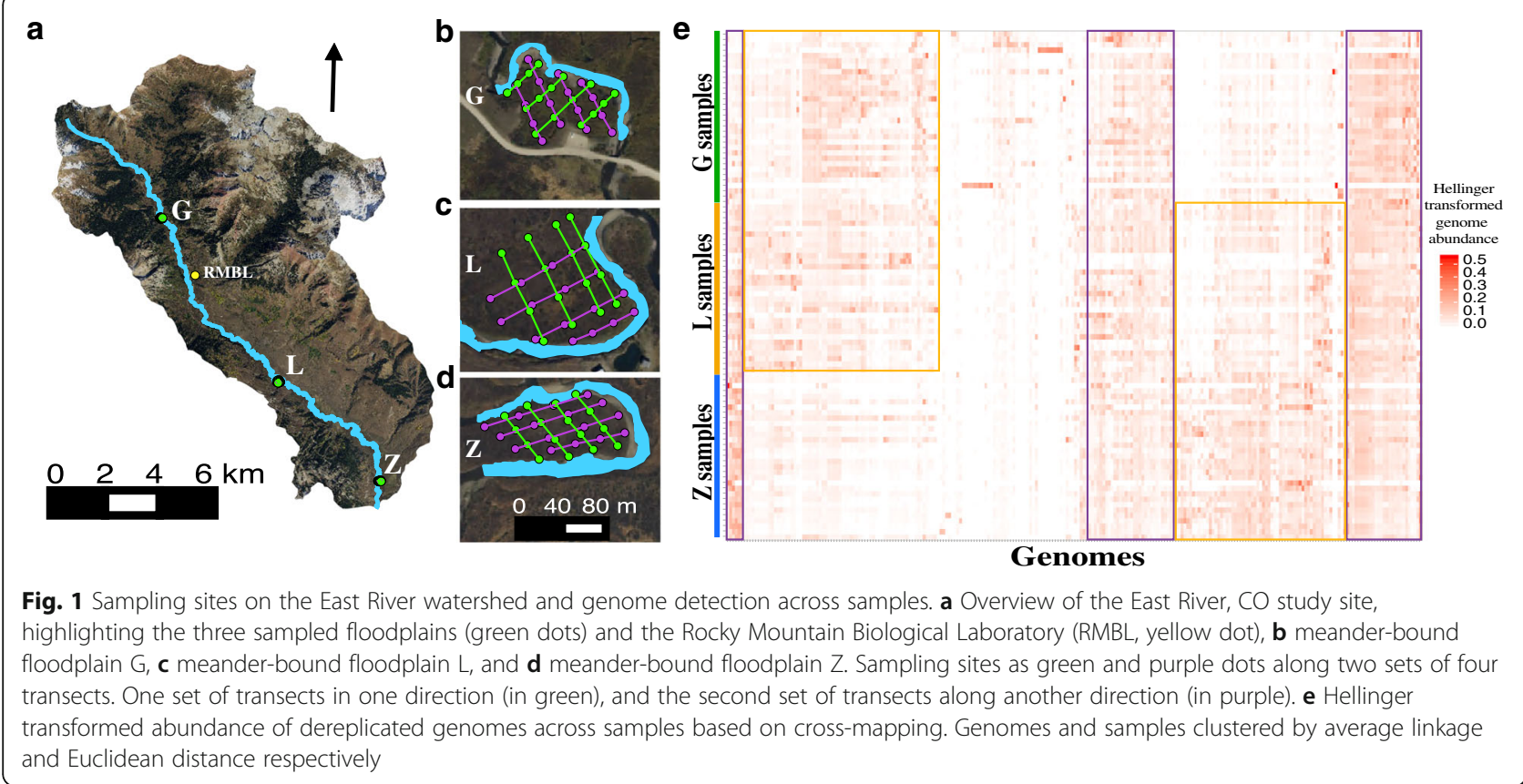

We constructed 1704 draft genomes from three floodplain datasets. About one third (622) of these genomes were classified as draft quality (237 from floodplain G, 150 from floodplain L, and 235 from floodplain Z). After dereplication at $99 \%$ average nucleotide identity (ANI) within each floodplain and correction of local assembly errors, we recovered 375 distinct genomes (173 from G, 94 from L, and 108 from Z). Dereplication across floodplains at $98 \%$ ANI generated a final set of 248 representative genomes for further analyses, predominantly $\geq 70 \%$ complete (Table S2, Additional file 2) and $46 \%$ of which were near-complete ( $\geq 90 \%)$.

We assessed our genome recovery effectiveness by comparing the number of genomes recovered to a secondary metric for quantifying unique species, the number of unique ribosomal protein L6 (rpL6) marker sequences within unbinned assemblies (see Methods section). The marker rpL6 has been shown to have high recoverability and species delineation accuracy [6], relative to methods such as full genome ANI. From the 94 metagenomes, we detected 930 distinct organisms based on rpL6 sequences clustered at $97.5 \%$ nucleotide identity. However, 571 of the distinct rpL6 sequences were on fragments with coverage that is too low for comprehensive genome sampling $(<7 \mathrm{X}$ coverage given our sequencing depth). The disparity relative to 248 reconstructed representative genomes relative to $359 \mathrm{rpL} 6$ on contigs at $>7 X$ coverage is attributed to significant challenges associated with genome recovery from soil (Figure S1, Additional file 3).

Candidate draft genomes were generated for almost all the organisms present at $>5-10 X$ coverage in each sample. However, on average, only $5.5 \%$ of the total read dataset was stringently mapped ( 2 mismatches per read of the pair) to the 248 genomes. This is not surprising, given that most sequencing allocations per sample were sufficient to genomically sample only organisms at $>\sim$ $0.25 \%$ relative abundance, and the most abundant organisms in each sample comprised only a few percent of the community.

In 2016, we returned to one of the floodplain sites (floodplain L) to collect samples for metatranscriptomics. We performed additional genomic sequencing from 19 of the original 32 sites (see Methods section; Table S1, Additional file 1) to provide a reference database for transcript mapping. These new DNA samples were sequenced at an average $3.7 \mathrm{Gbp}$ per sample (2.7$4.7 \mathrm{Gbp}$ ), for a total $\sim 0.2 \mathrm{Tbp}$ of sequencing. The RNA samples were sequenced at an average $10.8 \mathrm{Gbp}$ per sample $(3.2-15.1 \mathrm{Gbp})$ for a total of $\sim 0.15 \mathrm{Tbp}$ of sequencing. A total of 299 draft genomes were recovered from these samples, 123 of which passed our quality thresholds after curation. To examine stability across time, we pooled the 2015 and 2016 genome sets and dereplicated (at 95\% ANI) the combined set of 371 genomes, generating 215 genomes representative of distinct species. Notably, 32 species-level groups were detected in both years, and 29 were only detected in 2016 (Table S3, Additional file 4).

\section{Distribution of organisms within and across meander- bound floodplains}

To assess the presence of a representative genome in a sample, we relied on the sensitivity of read mapping to 
the dereplicated genome set. Based on our threshold for detection, about one-third of the genomes were from organisms that were consistently found across floodplains at similar levels of abundance (Purple boxes in Fig. 1 e). Regardless of their level of abundance, or which floodplain a representative genome was reconstructed from, the genomes were present in a median $>75 \%$ of the samples $(78 \%$ of upstream floodplain G samples, $84 \%$ of midstream floodplain L samples, and $87 \%$ of downstream floodplain Z samples; Figure S2a, Additional file 3). Additionally, the 248 organisms were present in the majority (median $88-91 \%$ ) of the other samples from the same floodplain from which the genome was reconstructed from (Figure S2b, Additional file 3).

Except for some genomes reconstructed from two floodplain G samples, the rest of the genomes were from organisms that shared more similar abundance levels if the floodplains were closer together within the river corridor (Yellow boxes in Fig. 1 e). More specifically, floodplains $\mathrm{G}$ and $\mathrm{L}$ or floodplains $\mathrm{L}$ and $\mathrm{Z}$ shared more organisms than floodplains $\mathrm{G}$ and $\mathrm{Z}$, which are located in the upper and lower reaches, respectively. Additionally, floodplain G is narrow, and is at times completely flooded, floodplain $\mathrm{L}$ is wider and may only flood partially, whereas floodplain $\mathrm{Z}$ is the widest and least prone to flooding.

Finally, we examined the number of samples where members of a 98\% ANI genome cluster were reconstructed from. The 248 genome clusters contained genomes reconstructed from between 2 and 39 samples. The largest genome set was for a large group of Betaproteobacteria strains generally related to strains detected in other environments such as soil, sediment, and water (Figure S3, Additional file 3; Data S1, Additional file 5). Genomes were reconstructed from two thirds of all samples from floodplain L. This result indicates that strains belonging to this Betaproteobacterial clade may play important roles in floodplain biogeochemistry (Fig. 2; Table

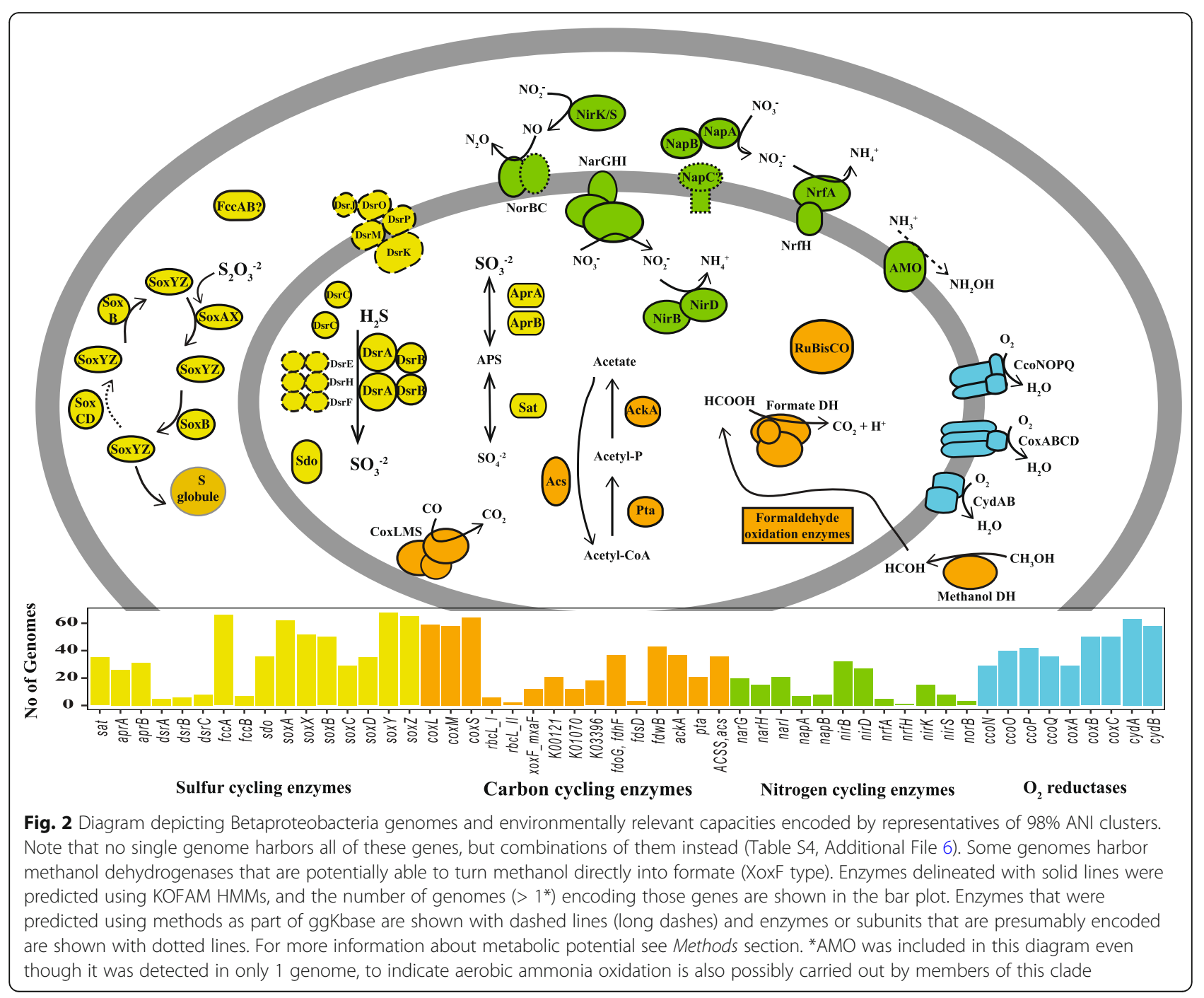


S4, Additional file 6), especially in soils associated with floodplain L.

\section{Taxonomic composition of the community}

Based on the 248 representative genomes detected in each sample, the phylum- or class-level community composition was broadly consistent both within and across floodplains (Fig. 3 a; Table S1, Additional file 1). This is also reflected in measures of alpha diversity between sites, as there was no significant difference in Shannon's diversity indices or unique number of organisms (Figure S4, Additional file 3). Some exceptions to this were Candidate Phyla Radiation (CPR) bacteria that seemed to be detected mostly in floodplain Z, while Thaumarchaeota seemed least present in this floodplain. Additionally, the number of the 248 genomes detected in each sample varied from sample to sample $(\mathrm{min}=35$ and $\max =212$ ). We detected particularly low numbers of genomes in five samples (T157 and T800 from floodplain
G and T133, T266, and T620 from floodplain Z), although only samples T133 and T266 from floodplain Z may have been affected by lower sequencing depths (Table S1, Additional file 1).

Betaproteobacteria was the group with the highest number of representative genomes (80) in all three floodplains. Other abundant taxa across floodplains included Deltaproteobacteria (27 representatives), Acidobacteria (21 representatives), Nitrospirae and Planctomycetes (both with 13 representatives), Gemmatimonadetes, Gammaproteobacteria, Chloroflexi, and Ignavibacteria (12, 11,11 , and 10 respectively).

\section{Uncovering a core floodplain microbiome}

To define a set of organisms representing a core floodplain microbiome, we identified organisms that were detected in most sampled sites ( $\geq 89$ of the 94 samples; 90th percentile), and whose abundance did not indicate a statistically significant enrichment in any specific

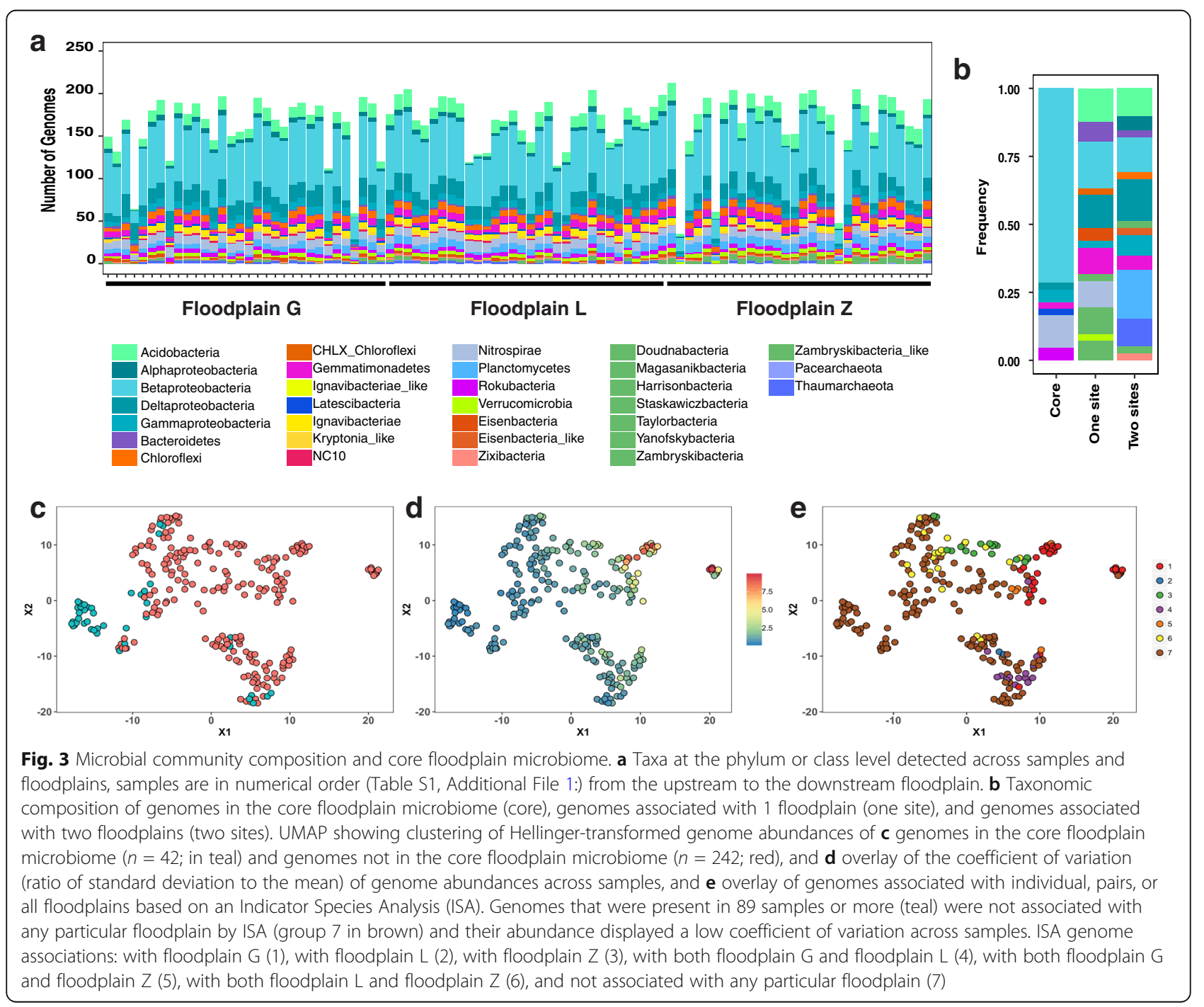


floodplain (by Indicator Species Analysis (ISA); see Methods section and Table S5, Additional file 7). This operational definition resulted in the identification of 42 high-prevalence organisms with low variance abundance profiles across all 3 meander-bound sites, which we refer to as the core floodplain microbiome (Fig. 3 c). In general, genomes with a low coefficient of variation of their abundance (blue dots in Fig. 3 d) overlapped with genomes that did not display a statistically significant association with any given floodplain (group 7, brown dots in Fig. 3 e), suggesting a wide distribution of these organisms across floodplains at similar abundance levels. The core floodplain microbiome was dominated by Betaproteobacteria, with lower abundances of Nitrospirae, Rokubacteria, Gemmatimonadetes, Gammaproteobacteria, Deltaproteobacteria, and Candidatus Letescibacteria (Fig. 3 b).

Genomes from organisms not considered to be part of the core floodplain microbiome were associated with one floodplain (G, L, or Z; $n=41$ ) or two floodplains ( $n$ = 39; Fig. 3 e). Other genomes were not classified as part of the core microbiome because although they were not statistically associated with one or two floodplains, they were not detected in $\geq 89$ samples $(n=126)$. Genomes affiliated with Acidobacteria, Bacteroidetes, and Chloroflexi were not part of the core floodplain microbiome and were associated with one or two floodplains. The ISA analysis supports the association of some CPR with one floodplain (i.e., between floodplain $\mathrm{Z}$ and Yanofskybacteria, Taylorbacteria, Harrisonbacteria, Staskawiczbacteria, and Zambryskibacteria-like bacteria). Similarly, bacteria in the Verrucomicrobia were associated with one floodplain (Z). Alphaproteobacteria, Thaumarcheota, Planctomycetes, other CPR (e.g., Zambryskibacteria and Doudnabacteria) and Eisenbacteria-like bacteria were associated with two floodplains.

\section{Geochemical functions, including those enriched in the core floodplain microbiome}

To determine what role floodplain soil Bacteria and Archaea may play in nutrient exports to the East River, we investigated a set of pathways involved in biogeochemical cycling and the microorganisms potentially responsible for them. The biogeochemical processes investigated include oxidation/reduction reactions associated with nitrogen, sulfur and hydrogen, $\mathrm{C} 1$ compound metabolism (e.g., $\mathrm{CO}_{2}$-fixation, $\mathrm{CO}$ oxidation, methanogenesis, methane oxidation, methanol oxidation, formate oxidation, methylamine oxidation, formaldehyde oxidation), $\mathrm{H}_{2}$ consumption or production, and the ability to use $\mathrm{O}_{2}$ as a terminal electron acceptor for aerobic respiration.

A set of Hidden Markov Models (HMMs) was used to annotate genes encoding for individual protein subunits that make up key enzymes and complete or partial metabolic pathways. For a given "function" (defined as the capacity to carry out a given biogeochemical transformation) to be encoded in a genome, certain criteria for presence had to be met (see Methods section; Table S4, Additional file 6). A total of 32 functions comprised the final set of biogeochemical transformations under investigation (Table S6, Additional file 8). It is important to note that in some cases, we also examined individual steps that are involved in a function, recognizing that some functions could be absent in a single genome because the pathway is carried out by multiple taxa (i.e., steps are encoded in multiple genomes). For example, denitrification occurs in separate steps involving different enzymes, and these steps can be performed by multiple different organisms. Complete ammonia oxidation, anaerobic ammonia oxidation, and methanogenesis (of any kind), were not detected in the dereplicated genome set, although some intermediary steps may still be ecologically relevant. Therefore, some steps involved in these pathways were included in downstream analyses.

To study the distribution of the functions of interest among genomes and across floodplains, we determined whether a function was present or absent in each genome in addition to where genomes were detected within and across floodplain samples. To describe the distribution of functions, we calculated the proportion of genomes with a given function compared with the total number of genomes detected in a sample. We found that the ability to use oxygen as an electron acceptor (aerobic respiration) was the most prevalent function among genomes (a median of 70$85 \%$ of genomes in each sample), followed by acetate metabolism (a median of 40-65\% of genomes in each sample), aerobic carbon monoxide (or other small molecule) oxidation, formate oxidation, and sulfide oxidation (a median of $30-50 \%$ of genomes in each sample; Fig. 4 a; Table S4, Additional file 6). This set of functions was consistently present across all three floodplains, whether encoded by the same or different taxa.

We also considered the distribution of functions that were detected in $<25 \%$ of genomes (Fig. 4 b-e; Table S4, Additional file 6). Of the remaining C1 transformations examined, methanol oxidation to formaldehyde was found in a median of $\sim 10 \%$ of the genomes. Of the sulfur transformations, sulfite $\left(\mathrm{SO}_{3}{ }^{-2}\right)$ oxidation to sulfate $\left(\mathrm{SO}_{4}{ }^{-2}\right)$, and thiosulfate $\left(\mathrm{S}_{2} \mathrm{O}_{3}{ }^{-2}\right)$ oxidation without sulfur $\left(\mathrm{S}^{0}\right)$ deposition and thiosulfate oxidation with sulfur deposition were most prevalent. For reactions involving hydrogen consumption or formation, genes encoding group $1 \mathrm{NiFe}$ hydrogenases (likely used for $\mathrm{H}_{2}$ oxidation) were found in a higher proportion of genomes than any other types of hydrogenases.

Nitrogen transformations were studied individually and as part of the nitrogen cycle. We found the capacity 


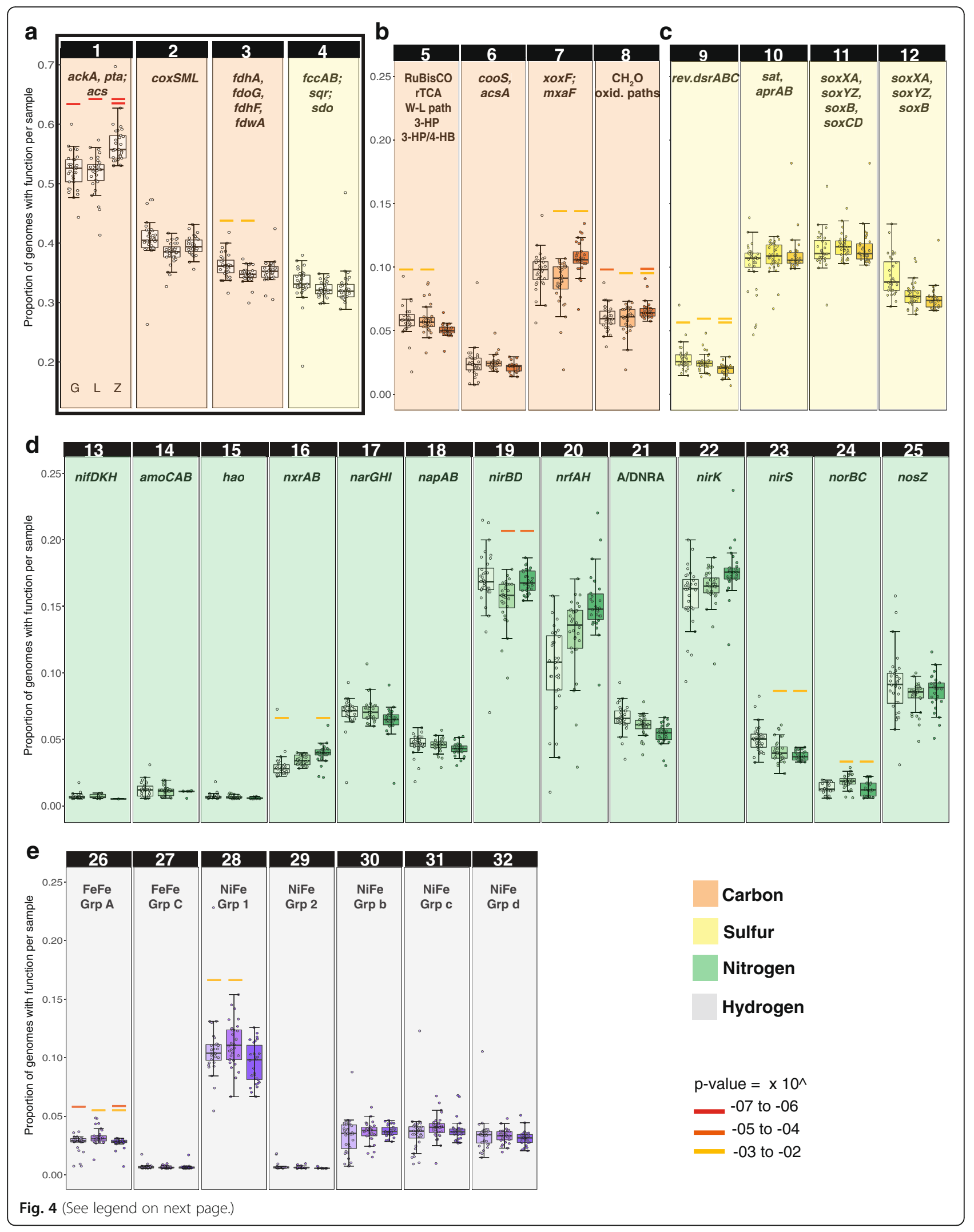




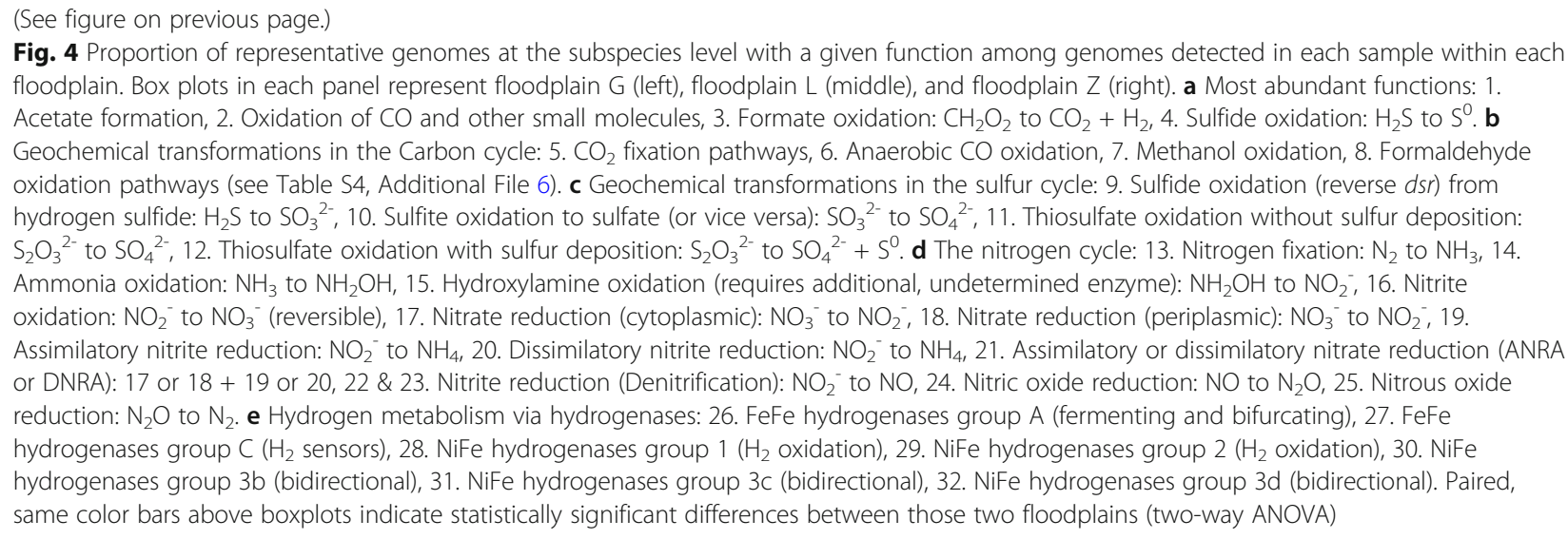

for nitrate $\left(\mathrm{NO}_{3}{ }^{-}\right)$use as a terminal electron acceptor in dissimilatory $\mathrm{NO}_{3}{ }^{-}$reduction in a substantially lower proportion of genomes $(2-10 \%)$ than the capacity to use $\mathrm{O}_{2}$ as a terminal electron acceptor. Of the reactions involved in nitrification, namely ammonia oxidation, hydroxylamine oxidation and nitrite $\left(\mathrm{NO}_{2}{ }^{-}\right)$oxidation, genomes encoding the oxidation of nitrite via nitrite oxidoreductase (NXR) were more common than genomes encoding the first two steps. The capacity for $\mathrm{NO}_{3}{ }^{-}$reduction as part of denitrification (NapAB or NarGHK) was encoded by far fewer genomes than $\mathrm{NO}_{2}{ }^{-}$reduction (which can be carried by via multiple enzymes, including NirK, NirS, NrfAH for dissimilatory nitrite reduction or NirBD for assimilation). Fewer genomes are predicted to encode the capacity to reduce nitric oxide (NO, the product of nitrite reduction) to nitrous oxide (via NorBC) than genomes with the capacity for nitrous oxide $\left(\mathrm{N}_{2} \mathrm{O}\right)$ reduction to $\mathrm{N}_{2}$. Overall, the most prevalent genomically encoded function was nitrite reduction, and capacities for consecutive nitrogen cycling steps were typically encoded in multiple different genomes. In other words, there is evidence to support the prevalence of metabolic handoffs [7] in the nitrogen cycle.

We identified functions that were significantly enriched (FDR $\leq 0.05$; hypergeometric test) in the core floodplain microbiome (a subset of ISA group 7) and found that the capacities to use $\mathrm{O}_{2}$ as a terminal electron acceptor, to perform aerobic $\mathrm{CO}$ or other small molecule oxidation, and thiosulfate oxidation (both with and without sulfur deposition) were enriched in these organisms.

\section{Environmental factors as drivers of function distribution across and within floodplains}

Environmental variables (Figure S5, Additional file 3) may explain in part the patterns of enrichment of genomically encoded functions described above. We first looked into correlations involving the following variables: total carbon (TC), total organic carbon (OC), total inorganic carbon (IC), total nitrogen (TN), organic carbon to nitrogen ratio (OC:N), distance of a sample to the river (Dist. to river), easting and northing (Cartesian coordinates for position on the floodplain), distance to the inner bank edge (from here on: toe distance) and distance to middle of the meander-bound floodplain as alternative measures of position on the floodplain (Figure S6, Additional file 3 ), topographic position index (TPI; as a proxy for the likelihood a site would be flooded during periods of high discharge or snowmelt), and elevation. Statistical analysis indicated that TC, OC, TN, and OC:N were all highly correlated with each other across the same set of metagenomic samples (Figure S7, Additional file 3 ), and their individual effects were not possible to disentangle. Thus, we chose either TC or OC for downstream analyses. Given the Northwest to Southeast orientation of the watershed, elevation, easting, and northing were all highly correlated with floodplain (G vs L vs Z), so only floodplain was included as a categorical variable. In summary, TC, floodplain, IC, TPI, distance to the river, and toe distance were the variables evaluated with the fourth corner method [8] to assess the response of each function at the gene level to the selected environmental or soil chemistry and GIS variables (see Methods section).

A group of biogeochemical transformations (gene level) displayed some correlation with environmental variables, particularly with individual floodplains (Fig. 5 a). Genome abundances were used as proxies for abundance of functions each genome encoded. The upstream floodplain G was positively correlated with thiosulfate oxidation (with and without $\mathrm{S}$ deposition), sulfite oxidation, sulfide oxidation, $\mathrm{O}_{2}$ as a terminal electron acceptor, aerobic $\mathrm{CO}$ or other small molecule oxidation, and acetate metabolism. Only $\mathrm{N}_{2} \mathrm{O}$ reduction was positively correlated with the middle floodplain L. The downstream floodplain $\mathrm{Z}$ was positively correlated with $\mathrm{H}_{2}$ oxidation via group $1 \mathrm{NiFe}$ hydrogenases (a function that was negatively correlated with upstream floodplain G). Most sulfur compound 


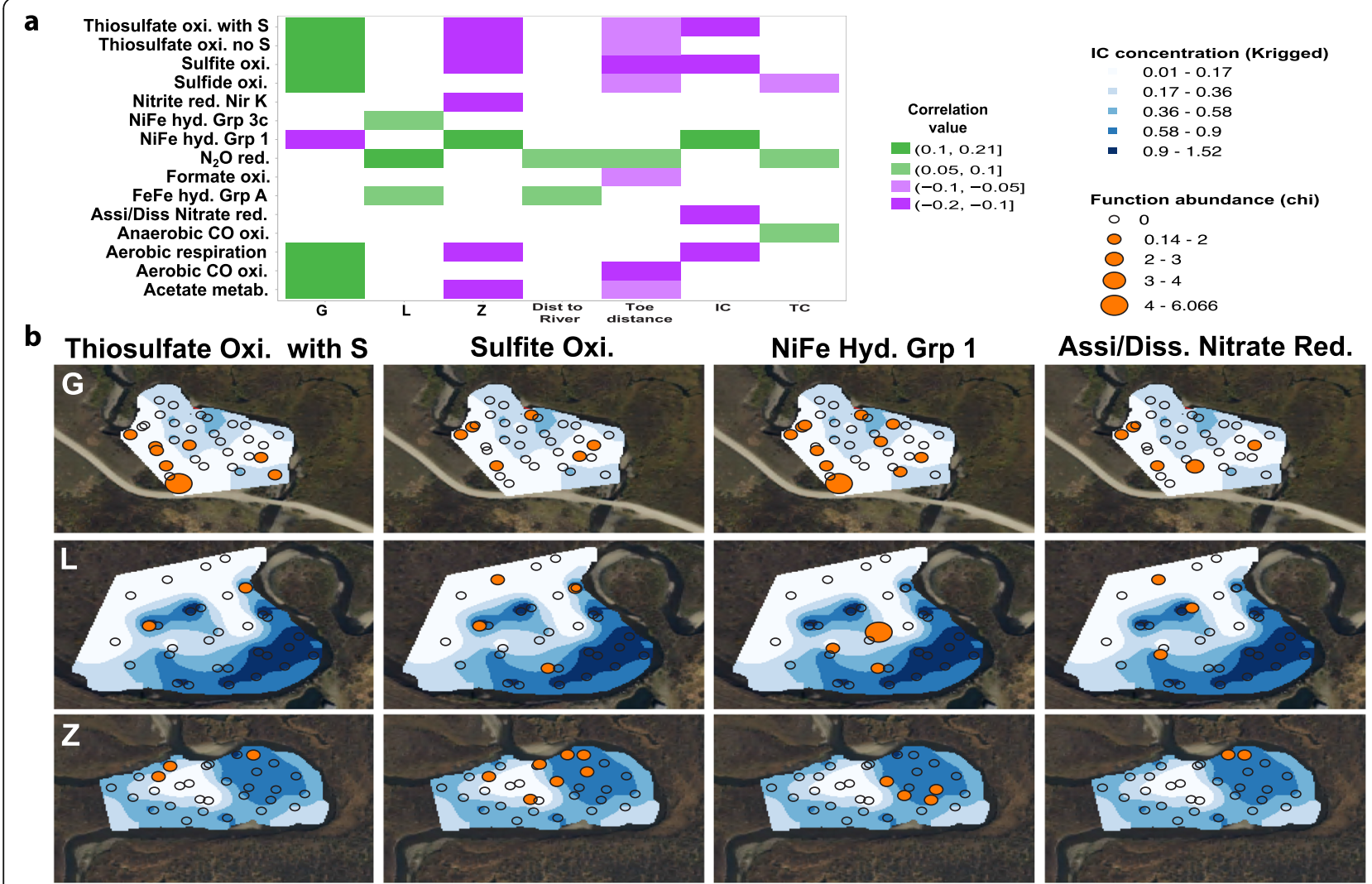

Fig. 5 Function abundance and its correlation with environmental variables. a Significant positive (green) or negative (violet) correlations between environmental variables (bottom) and biogeochemical transformations (left) identified by a fourth corner analysis. b Abundance of genomes encoding functions positively correlated with inorganic carbon concentrations (IC; \%)

transformations, as well as nitrite reduction, aerobic respiration and acetate metabolism were negatively correlated with floodplain Z (Fig. 5 a).

Overall, genomes with the capacity for aerobic respiration and sulfur compound oxidation are most prevalent towards the headwaters (floodplain G), and within this floodplain, sulfur compound oxidation apparently is associated with low IC (Fig. 5 b). Within the downstream meander where aerobic respiration is least prominent in the genomes, bacteria able to oxidize $\mathrm{H}_{2}$ via Group 1 NiFe hydrogenases appear correlated with somewhat elevated concentrations of IC (Fig. 5 b).

\section{Potentially active genes encoding key biogeochemical transformations in the riparian zone}

To determine whether key functions encoded in the genomes were transcriptionally active at the time of sampling (early September 2016; during base flow conditions like previous year), we resampled floodplain L for metatranscriptomics and metagenomics. This floodplain was chosen among the three because it shared the majority of organisms detected in 2015 with the other two floodplains.
Considering potential differences between the 2 years, metatranscriptomic reads were mapped to a dereplicated genome set at the species level (95\% ANI), which comprised 215 genomes reconstructed from samples collected in 2015 and 2016. We calculated transcript counts using read pairs mapped to predicted open reading frames (ORFs) with at least 95\% nucleotide identity (see Methods section). The highest median transcript counts were observed for Nitrospirae and Betaproteobacteria, followed by Candidatus Latescibacteria and Eisenbacteria-like bacteria, Rokubacteria, and Deltaproteobacteria (Fig. 6 a; Data S2, Additional file 9). We also evaluated the number of reads mapping to genes encoding key functions and determined what percentile in the distribution of transcription levels each gene fell in.

Key genes involved in potentially active biogeochemical transformations with a median transcription $>75$ th percentile of all the genes transcribed in a given genome included $a m o C A B$, and $n x r A B$, involved in nitrification. The amoCAB genes for aerobic ammonia monooxygenase were found in the 90th percentile of transcribed genes across genomes. However, these genes were present in very few genomes (one Nitrospirae and two Thaumarcheota). Similarly present in few genomes and 


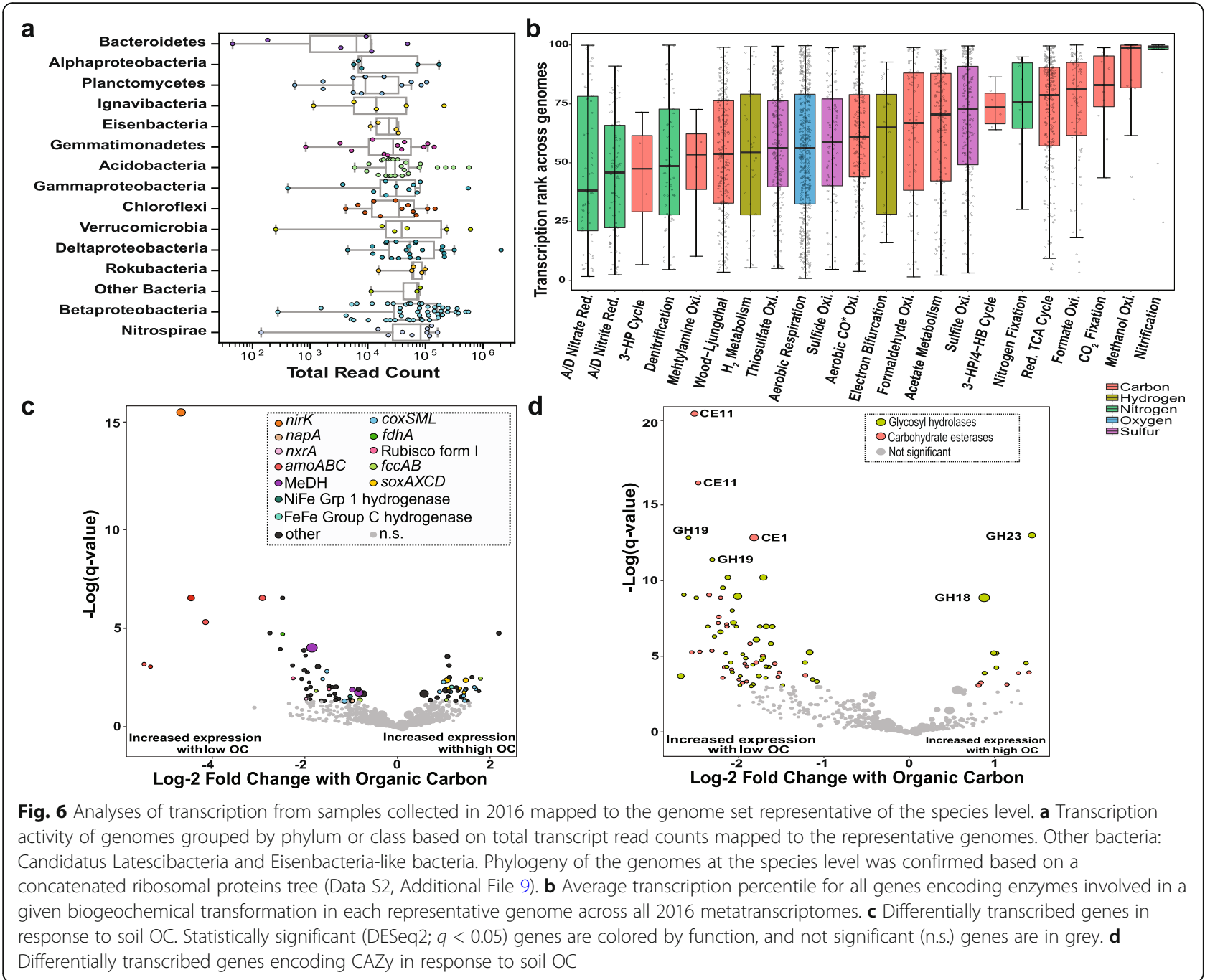

also highly transcribed were genes involved in $\mathrm{CO}_{2}$ fixation, specifically $\mathrm{RuBisCO}$ forms I and II and enzymes in the reductive TCA cycle (OFOR and citrate lyase). Other highly transcribed genes were for methanol oxidation to formaldehyde ( $x o x F, m x a F$ ) and formate oxidation ( $f d h A B, f d o G, f d h F, f d w A, f d s D, f d w B)$ as part of $C 1$ metabolism (Figure S8, Additional file 3 ).

Functions enriched in the core floodplain microbiome (aerobic $\mathrm{CO}$ or other small molecule oxidation, thiosulfate oxidation, and the ability to use $\mathrm{O}_{2}$ as a terminal electron acceptor via $\operatorname{cox} A B C D, c y d A B$ or $c c o N)$ and functions that displayed some degree of correlation with environmental variables in gene abundance (e.g., sulfite oxidation via sat and $a p r A B$ or $\operatorname{dsr} A B$ and sulfide oxidation via $f c c A B$ ) were most often between the 50th and 75th percentiles of transcribed genes per genome. Surprisingly, given their prominence in genomes, genes involved in nitrogen cycling such as narGHI or napA and especially $n r f A H$ responsible for dissimilatory nitrite reduction, nirK and nos $Z$ responsible for some denitrification steps, displayed transcription levels only between the 35th and 50th percentiles (Fig. 6 b).

We tested for differential transcription levels in response to changes in environmental variables (Table S7, Additional file 1) using DESeq2 [9]. Of the four environmental variables that were highly correlated with each other (TC, OC, TN, OC:N; either positively or negatively (Figure S9, Additional file 3), we observed the strongest differential gene expression in response to OC (Fig. 6 c). In samples with higher concentrations of $\mathrm{OC}$, genes involved in the Sox pathway for thiosulfate oxidation (soxAX and soxCD) and those involved in aerobic CO or other small molecule oxidation (coxLMS) were highly transcribed. More specifically, transcripts mapped to one coxL form I gene (true carbon monoxide dehydrogenase, $\mathrm{CODH}$ ) and the rest mapped to four other coxL form II 
genes (carbon monoxide-like dehydrogenase). The form I transcripts were correlated with high $\mathrm{OC}$, and the form II transcripts with both low (1 hit) and high OC (3 hits).

In samples with low OC, highly transcribed genes included those involved in methanol oxidation ( $x \circ x F$, $m x a F)$, formate oxidation $(f d h A)$, sulfide oxidation $\left(f_{c c} A B\right)$, hydrogen metabolism (NiFe Grp1), ammonia oxidation $(a m o A B C)$, nitrite oxidoreduction $(n x r A)$, nitrate reduction (napA), and nitrite reduction (nirK). Samples with low OC also have low TN, which may in part be attributed to high activity of microbial nitrification followed by denitrification, with denitrification reliant on consumption of OC.

As might be expected, RuBisCO form I was highly transcribed under conditions of low OC. Activity of the Calvin Benson Bassham (CBB) pathway for $\mathrm{CO}_{2}$ fixation is linked to Betaproteobacteria, Deltaproteobacteria, Gammaproteobacteria, and NC10, some of which have metabolisms fueled by oxidation of intermediate sulfur compounds (e.g., sulfide or thiosulfate oxidation). The observations reveal a potentially important source of organic carbon in some floodplain soils. In terms of overall transcriptional activity, autotrophic pathways may not be expressed but the organisms may otherwise be highly transcriptionally active. In fact, mostly organisms from the phyla Thaumarchaeota, Rokubacteria, NC10, and Nitrospirae were active under low OC conditions. Betaproteobacteria and Acidobacteria were transcriptionally active under conditions of higher OC.

We also investigated the potential for organic matter degradation through transcription of genes encoding carbohydrate-active (CAZy) enzymes (Table S8, Additional file 11) [10]. We narrowed our search to CAZy enzyme types that were present in at least $60 \%$ of the genomes, as a proxy for widespread distribution in the floodplain soil microbial community. The most abundantly transcribed CAZy genes were in the glycosyl hydrolase $(\mathrm{GH})$ and carbohydrate esterase $(\mathrm{CE})$ classes. In general, the highly transcribed enzymes in the $\mathrm{CE}$ class use hemicellulose and amino sugars as substrates, resulting in acetate as a byproduct. Acetate could be utilized by many floodplain organisms, considering the prevalence of genes involved in acetate metabolism among the genomes. Enzymes in the GH class use cellulose, pectin, chitin, and starch as substrates, releasing a variety of sugars as byproducts, which can be utilized for central metabolism during growth. We then tested for CAZy differential expression in response to changing concentrations of organic carbon. Twelve CAZys (both $\mathrm{GH}$ and CEs) expressed by three strains of Betaproteobacteria increased in expression in samples with high OC. Many of the same classes of GH and CE displayed high levels of transcription correlated with both low and high OC levels (e.g., GH23, GH28, CE4, and CE11; Fig. 6 d), although gene expression by three strains of Betaproteobacteria correlated with high levels of OC. Similar CAZy enzymes were expressed by NC10, Nitrospirae, and Rokubacteria, the same organisms commonly associated with high levels of transcription under low OC.

\section{Discussion}

Biogeochemical processes modulate $\mathrm{C}, \mathrm{S}$, and $\mathrm{N}$ exports from watersheds, including the East River [11]. Important questions relate to the sources and sinks of these compounds and the biological controls on them. Some data indicate that a subset of the organic carbon in sediments from East River floodplains derives from the shale, although plants are the obvious central source for fixed carbon in areas of more developed soils [12]. $\mathrm{CO}_{2}$ fixation genes were relatively rarely detected in the bacterial genomes, which might be interpreted to support this deduction. However, genes for $\mathrm{CO}_{2}$ fixation in a few organisms were very highly transcribed, indicating at least periodic inputs of microbially produced organic carbon into riparian zone soils. Spatially, high activity of genes involved in $\mathrm{CO}_{2}$ fixation was correlated with low organic carbon concentrations in soil. Many organisms predicted to rely on $\mathrm{CO}_{2}$ fixation as their main carbon source are aerobic chemolithoautotrophs that oxidize inorganic compounds (e.g., $\mathrm{NH}_{3}^{+}, \mathrm{NO}_{2}^{-}, \mathrm{S}^{0}, \mathrm{H}_{2} \mathrm{~S}, \mathrm{H}_{2}, \mathrm{CO}$, $\mathrm{S}_{2} \mathrm{O}_{3}$ ) as a source of energy. Thus, we infer significant linkages among these key element nutrient cycles.

Low concentration of organic carbon also correlated with high activity of genes involved in methanol oxidation. Methanol results from the breakdown of plant material, such as pectin and lignin [13, 14], and the activity of methanol dehydrogenases may be indicative of decomposed organic matter. Similarly, low concentration of organic carbon correlated with high activity of genes involved in sulfide and $\mathrm{H}_{2}$ oxidation, nitrification, and interconversion of nitrite and nitric oxide. The organisms responsible for these reactions are primarily autotrophs.

Interestingly, the capacities for thiosulfate oxidation/ elemental sulfur formation, sulfite oxidation and $\mathrm{H}_{2}$ oxidation, as well as assimilatory or dissimilatory nitrate reduction to ammonia (ANRA or DNRA) were patchily spatially distributed (Fig. 5 b), possibly localized by lower inorganic carbon concentrations. Further, genes for sulfur compound oxidation were more prominent in genomes of organisms from the upstream floodplain, which is closer to the adjoining hill, possibly reflecting higher inputs of intermediate sulfur compounds from rock weathering reactions in the headwater compared with downstream regions. The sources of thiosulfate could be weathering of detrital grains of shale-associated pyrite and/or reoxidation of microbially produced sulfide in anoxic OC-rich regions of the soil or underlying river 
sediment. Closer proximity to igneous intrusives in the upstream part of the drainage (e.g., near Floodplain G) leads to greater incidence of pyrite-bearing shales. It has been shown previously that hydrological connectivity can shape microbial activity, with low connectivity linked to higher abundance of genes involved in sulfur metabolism [15]. In the East River, sulfur compounds may be redistributed from upstream to downstream regions, but the degree of hydrologic connectivity within and across floodplains is uncertain and varies dramatically over the course of the year [16]. Additionally, these shallow soils may only be hydrologically connected to the river during high water flood events or through vertical transport.

By contrast, high OC levels correlated with high activity of genes involved in oxidation of CO (form I CODH), and other small carbon compounds (form II or other subtypes), which may be substrates for carbon monoxide dehydrogenases [5]. CO may be sourced from the atmosphere, by thermochemical, photochemical, and chemical degradation of organic matter in soils and marine sediments, and from biological production by microbes, leaves, roots, and animals [17]. East River CO oxidizers are most likely carboxydovores that require organic carbon to grow, even though they can oxidize $\mathrm{CO}$ at atmospheric levels (i.e., they use a high affinity form I CODH) $[18,19]$. This is in contrast to carboxydotrophs that grow with $\mathrm{CO}$ as the sole energy and carbon source and require $\mathrm{CO}$ at greater than atmospheric concentrations (for a low affinity form I carbon monoxide dehydrogenase (CODH) [17]). Additionally, form II CO dehydrogenases seem to play a key role in this ecosystem, although very little is known about their actual function. Detection of a high prevalence of $\mathrm{CODH}$ and $\mathrm{CODH}$-like enzymes echoes results from a grassland soil system, a study by Diamond et al. [5], reinforcing the suggestion that small carbon compounds such as plant exudates, may be an important carbon currency under some conditions.

High organic carbon levels also correlated with high activity of genes involved in thiosulfate oxidation, and carbohydrate esterases and glycosyl hydrolases such as GH23 (lysozyme) and GH18 (chitinase). These GHs would be required for organic matter degradation at locations of higher carbon availability, where presumably plants and fungi are more abundant. Bacteria that degrade plant biomass are also known to employ catabolite repression of CAZy enzymes [20], perhaps explaining the lower diversity of CAZys under these conditions. Different variants of these carbohydrate-active genes were highly expressed in a variety of taxa including Rokubacteria, Nitrospirae, and NC10 in soil with low organic carbon, where diverse carbon sources must be exploited for survival.

Many watershed ecosystems are limited by access to biologically available nitrogen, the important sources of which are likely to be shale bedrock weathering [21], atmospheric deposition [22], and nitrogen fixation. A complex interplay of biological processes impact nitrogen speciation and bioavailability, including ammonia oxidation (nitrification), denitrification to $\mathrm{N}_{2}$, and nitrite assimilation via ANRA or DNRA. The nitrogen budget can be addressed by direct measurement of inputs, plant-associated inventories, and the concentration of inorganic and organic nitrogen compounds exported from the watershed via rivers [23, 24]. By comparing these numbers, it may be possible to estimate the fraction of the bioavailable nitrogen that is lost from the system via loss as $\mathrm{N}_{2}$ and trace gases. What is missing from this analysis is an estimate of the degree to which nitrogen compounds are recycled, the role of riparian zone soils in these processes, and the potential for subsurface storage of nitrogen compounds in microbial biomass.

Using genome-resolved metagenomics, we identified the capacity for nitrogen fixation and ammonia oxidation to nitrite and nitrate in relatively few organisms, yet the metatranscriptomic data show these to be highly active functions. Thus, we infer important microbial contributions to reservoirs of oxidized nitrogen compounds in riparian zone soils, with the potential to substantially augment inputs from atmospheric deposition and bedrock weathering. Genes involved in nitrite reduction (dissimilatory nitrate reduction to ammonium or denitrification to $\mathrm{N}_{2}$ ), while abundant in comparison to other capacities for nitrogen transformation, displayed surprisingly low levels of transcription at the time of sampling. Over the course of the year, the fluctuating water table and periodic flooding should provide environmental niches for both obligately aerobic, and anaerobic processes. Furthermore, the soil oxidation state and the carbon to nitrate ratio, particularly in nitrogen-limited systems, may favor DNRA over denitrification [25]. The current study was conducted during base flow conditions (both years), well after the snowmelt season, when high river discharge induces flooding and therefore anoxic conditions. In the meanderbound floodplains, snowmelt-derived flow in this ecosystem persists well into the year [12], so shallow soils may be flooded long after discharge levels drop. The results raise the possibility of coupling of nitrification and dissimilatory nitrate pathways on a temporal basis, under baseflow conditions (when nitrification is dominant), or under snowmelt conditions (when dissimilatory processes occur). High net nitrification has been reported for riparian zones when the water table is below $-30 \mathrm{~cm}$ [26], accordingly the water table in floodplain L was observed to be below this level in September 2016. Overall, reassimilation of nitrogen as ammonium may be important in this ecosystem, particularly if nitrogen limited.

An important result from the current study was that there appears to be a core floodplain microbiome composed 
of specific bacterial species from Betaproteobacteria, Gammaproteobacteria, Deltaproteobacteria, Nitrospirae, Candidatus Latescibacteria, and Rokubacteria; and all of these groups were transcriptionally active at the time of sampling. Many of the clusters of related genomes are relatively distantly related to previously described bacterial types. Thus, we conclude that many of the most abundant taxa in these riparian zone soils are organisms that have, until now, remained essentially outside of the range of scientific investigations. Importantly, capacities for aerobic respiration, aerobic oxidation of $\mathrm{CO}$ and other small molecules, as well as thiosulfate oxidation with formation of elemental sulfur, were enriched in the core floodplain microbiome. Notably, the most abundant functions of the core microbiome were only moderately transcribed at the time of sampling.

The current study uncovered functions that are widely encoded in genomes across all three floodplains and assayed in situ microbial activity. The findings motivate extensive detailed chemical characterization, particularly of one carbon compounds, ammonia and other nitrogen and sulfur species so that it will be possible to link microbial activity to fluxes of nutrients from the riparian zone. Our interpretations regarding biogeochemical cycling of $\mathrm{C}, \mathrm{N}, \mathrm{S}$, and $\mathrm{H}$ were based on the set of reconstructed genomes from the floodplain soils. It is certainly true that this may have made some patterns harder to detect than might have been the case with more comprehensive analyses. Importantly, however, we were able to recover genomes with widespread distribution across the soils and to incorporate into our analyses organisms that were much less abundant, but transcriptionally active. In general, we found that gene and organism abundances do not predict transcription levels. The in situ transcription data revealed the potentially very high importance of rare genes and organisms. However, it is important to note that transcript datasets are a snapshot from a moment in time, and that transcription patterns will vary across seasons and maybe even daily. Notably, our analyses showed organismal and functional overlap in microbial communities found both within and across the three floodplains over two consecutive years $(\sim 15 \%$ of species in common, despite the very high diversity and complexity of the soils). Thus, in contrast to potentially substantial transcriptome variability, gene inventories reflect metabolic potential that likely remains fairly constant throughout the year.

\section{Conclusions}

This study employed deep sequencing and genomeresolved metagenomics, in combination with geochemistry, to document commonalities among three similar but geographically distant meander-bound floodplain ecosystem compartments. We report the existence of microbial strains that potentially perform similar biogeochemical functions across these compartments. This is important, given that soil microbial communities are exceedingly complex and potentially very heterogeneous. We conclude that, at the watershed scale, meander-bound regions of floodplain soils can be defined as "functional zones" that likely predict biogeochemical transformations along the riparian corridor, thereby providing broadly generalizable inputs to ecosystem models.

\section{Methods}

\section{Study site and sample collection}

The East River (ER) watershed has been described elsewhere [3]. In brief, the ER watershed is a $300-\mathrm{km}^{2}$ area largely underlain by marine shales of the Cretaceous Mancos formation located in the Elk Mountains in westcentral Colorado. The ER is a headwaters catchment in the Upper Colorado River basin, with an average elevation of $3350 \mathrm{~m}$. At about 62-km long, the ER traverses an elevational gradient that includes alpine, subalpine, and montane life zones as a function of stream reach. The average annual temperature is $\sim 0{ }^{\circ} \mathrm{C}$, with long cold winters and short cool summers, and the majority of precipitation is received in the form of snow [27].

The sampling sites are located across an altitudinal gradient followed by the river $(\sim 2700-2900 \mathrm{~m})$. The floodplain at the highest elevation is located ca. $6 \mathrm{~km}$ from the headwaters, nearby Gothic, Colorado, site of the Rocky Mountain Biological Laboratory (RMBL, Fig. 1). Therefore, samples collected from this site were named East River Meander-bound floodplain G (ERMG). The second site was located ca. $8 \mathrm{~km}$ downstream of Gothic, among a series of floodplains, one of which is situated adjacent to an intensive research site of the Watershed Function SFA [3]. This floodplain stands out because of its larger size, and samples were named ERML (L for large). The third site was located ca. $18 \mathrm{~km}$ downstream of Gothic and just upstream of the confluence with Brush Creek. Samples from this site were named ERMZ, with the stream reach between ERML and ERMZ being characterized by a relatively low gradient with high sinuosity.

In September 2015, during base flow conditions, two series of perpendicular transects were laid out at each site. Each set of transects comprised four transects that were parallel between them (Fig. 1). One set of transects were approximately North to South (T1-T4), and the other set of transects were East to West (T5-T8). The starting point of each transect was designated " $0 \mathrm{~m}$ ", and the location of the other sites along the transect was relative to the point of origin. A Trimble Geo 7X GPS was used to determine the exact location of each site along the transects with an accuracy of $0.5 \mathrm{~m}$. The distance (in meters) of each sample to the point of origin was included in the sample name, which comprised the 
initials of the study area (ER), the initials for each meander-bound floodplain (i.e., MG, ML, or MZ), the transect number (i.e., T1-T8), and the distance in meters from the first sample collected at the start point (e.g., $19 \mathrm{~m}$ ). We sampled areas of $\sim 4600 \mathrm{~m}^{2}$ in floodplain $\mathrm{G}, \sim 8000 \mathrm{~m}^{2}$ in floodplain $\mathrm{L}$, and $\sim 5400 \mathrm{~m}^{2}$ in floodplain Z.

Four soil samples from the $10-25 \mathrm{~cm}( \pm 1-2 \mathrm{~cm})$ soil depth interval were collected in the span of 10 days along each one of the eight transects, for a total of 32 samples per floodplain. Each site was cleared of grasses and other vegetation with clippers, and the first $\sim 10 \mathrm{~cm}$ of soil was removed with a sterile shovel. Soil samples were collected using sterile tools, including a soil core sampler and $7.6 \times$ $15.2 \mathrm{~cm}$ plastic corer liners (AMS, Inc), stainless-steel spatulas, and Whirl-pak bags. Samples were immediately stored in coolers for transportation to RMBL, where samples were prepared for archival and transportation to the University of California, Berkeley. Soil cores were broken apart and manually homogenized inside the Whirl-pak bags. Subsamples for chemical analyses, DNA extractions, and long-term archival were obtained inside a biosafety cabinet, kept at $-80^{\circ} \mathrm{C}$, transported in dry ice, and stored at $-80^{\circ} \mathrm{C}$ at the University of California, Berkeley.

In September 2016, another round of sample collection was conducted at floodplain L for metagenomics, metatranscriptomics, and chemical analyses. A subset of 19 out of the 32 sampling sites from the previous year was targeted, and a subset (15) of those was also selected for metatranscriptomics (Table S1, Additional file 1). Given that floodplain L was the site with the lowest total number of draft genomes recovered in 2015, we added new sites closer to the original sites with the intent of increasing this number by leveraging differential coverage across samples [28]. Four new sites located in between the original transects (denominated ERMLIBT) and two sites adjacent to ERMLT660 (ERMLT660_1 and ERML T660_2) were sampled. Additionally, samples were collected from above the water table (approximately below $40-50 \mathrm{~cm}$ from the surface) at a depth of 32-47 $\mathrm{cm}( \pm 4-6 \mathrm{~cm})$ from three sites (ERMLT200, ERML231, and ERML293) along T2. Samples from the 11-25 cm $( \pm 1-1 \mathrm{~cm})$ soil layer were obtained following the same protocol as the previous year, with the exception that subsamples for RNA sequencing were preserved in situ. Once the soil cores were transferred to a Whirl-pak bag, they were manually homogenized inside the bags. Eight grams $(8 \mathrm{~g})$ of soil were collected using sterile stainlesssteel spatulas directly into $50-\mathrm{mL}$ sterile falcon tubes containing $20 \mathrm{~mL}$ of LifeGuard Soil Preservation Solution (Qiagen) for RNA preservation. The samples were mixed by hand to saturation with the LifeGuard solution, stored in a chilled cooler for transportation to RMBL and later stored at $-80{ }^{\circ} \mathrm{C}$.

\section{Soil chemistry}

Total carbon (TC) and total inorganic carbon (TIC) were analyzed using a Shimadzu TOC-VCPH analyzer equipped with a solid sample module SSM-5000A (Shimadzu Corporation, Japan). Total organic carbon (TOC) was obtained from the difference between TC and TIC. For TC quantification, a subsample of the dried solids was weighed into a ceramic boat and combusted in a TC furnace at $900{ }^{\circ} \mathrm{C}$ with a stream of oxygen. To ensure complete conversion to $\mathrm{CO}_{2}$, the generated gases are passed over a mixed catalyst (cobalt/platinum) for catalytic post-combustion. The $\mathrm{CO}_{2}$ produced is subsequently transferred to the NDIR detector in the main instrument unit (TOC-VCSH). Quantification of the inorganic carbon was carried out in a separate IC furnace of the module. Phosphoric acid is added to the sample, and the resulting $\mathrm{CO}_{2}$ is purged at $200{ }^{\circ} \mathrm{C}$ and measured.

Total nitrogen (TDN) was analyzed using a Shimadzu Total Nitrogen Module (TNM-1) coupled to the solid sample module (SSM-5000A) and TOC-VCSH analyzer (Shimadzu Corporation, Japan). TNM-1 is a nonspecific measurement of TN. All nitrogen species in samples were combusted at $900{ }^{\circ} \mathrm{C}$, converted to nitrogen monoxide and nitrogen dioxide, then reacted with ozone to form an excited state of nitrogen dioxide. Upon returning to the ground state, light energy is emitted. Then, TN is measured using a chemiluminescence detector.

\section{DNA extraction and sequencing}

Genomic DNA was extracted from $\sim 10 \mathrm{~g}$ of thawed soil using Powermax Soil DNA extraction kit (Qiagen) with some minor modifications as follows. Initial cell lysis by vortexing vigorously was substituted by placing the tubes in a water bath at $65{ }^{\circ} \mathrm{C}$ for $30 \mathrm{~min}$ and mixing by inversion every $10 \mathrm{~min}$ to decrease shearing of the genomic DNA. After adding the high concentration salt solution that allows binding of DNA to the silica membrane column used for removal of chemical contaminants, vacuum was used instead of multiple centrifugation steps. Finally, DNA was eluted from the membrane using 10 $\mathrm{mL}$ of the elution buffer (10 $\mathrm{mM}$ Tris buffer) instead of $5 \mathrm{~mL}$ to ensure full release of the DNA. DNA was precipitated out of solution using $10 \mathrm{~mL}$ of a $3-\mathrm{M}$ sodium acetate (pH 5.2) and glycogen $(20 \mathrm{mg} / \mathrm{mL})$ solution and $20 \mathrm{~mL} \mathrm{100 \%} \mathrm{sterile-filtered} \mathrm{ethanol.} \mathrm{The} \mathrm{mix} \mathrm{was} \mathrm{incu-}$ bated overnight at $4{ }^{\circ} \mathrm{C}$, centrifuged at $15,000 \times g$ for 30 min at room temperature, and the resulting pellet was washed with chilled $10 \mathrm{~mL}$ sterile-filtered $70 \%$ ethanol, centrifuged at $15,000 \times g$ for $30 \mathrm{~min}$, allowed to air dry in a biosafety cabinet for $15-20 \mathrm{~min}$, and resuspended in $100 \mu \mathrm{L}$ of the original elution buffer. Genomic DNA yields were between 0.1 and $1.0 \mu \mathrm{g} / \mu \mathrm{L}$ except for two samples with $0.06 \mu \mathrm{g} / \mu \mathrm{L}$. Power Clean Pro DNA clean up kit (Qiagen) was used to purify $10 \mu \mathrm{g}$ of DNA 
following manufacturer's instructions except for any vortexing which was substituted by flickering of the tubes to preserve the integrity of the high-molecular-weight DNA. DNA was resuspended in the elution buffer $(10$ $\mathrm{mM}$ Tris buffer, $\mathrm{pH} 8$ ) at a final concentration of $10 \mathrm{ng} /$ $\mu \mathrm{L}$ and a total of $0.5 \mu \mathrm{g}$ of genomic DNA. DNA was quantified using a Qubit double-stranded broad range DNA Assay or the high-sensitivity assay (ThermoFisher Scientific) if necessary. Additionally, the integrity of the genomic DNA was confirmed on agarose gels and the cleanness of the extracts tested by absence of inhibition during PCR. For samples collected the following year, DNA was co-extracted with RNA (see next section), in addition to extracting subsamples (10 $\mathrm{g}$ of soil) from the same core following the extraction protocol described above (Table S1, Additional file 1).

Clean DNA extracts and co-extracts were submitted for sequencing at the Joint Genome Institute (Walnut Creek, CA), where samples were subjected to a quality control check. Two of the 96 samples from 2015 failed QC and thus were not sequenced (ERMZT233 and ERMZT446), and four samples were sequenced ahead of the others (ERMLT700, ERMLT890, ERMZT100, and ERMZT299). Ten out of 15 of the DNA co-extracts from 2016 failed QC due to low DNA yields and were not sequenced either. Sequencing libraries for the first four samples were prepared in microcentrifuge tubes. One hundred nanograms of genomic DNA was sheared to $600 \mathrm{bp}$ pieces using the Covaris LE220 and size selected with SPRI using AMPureXP beads (Beckman Coulter). The fragments were treated with end repair, A-tailing, and ligation of Illumina-compatible adapters (IDT, Inc) using the KAPA Illumina Library prep kit (KAPA biosystems). Libraries for the rest of the samples were prepared in 96-well plates. Plate-based DNA library preparation for Illumina sequencing was performed on the PerkinElmer Sciclone NGS robotic liquid handling system using Kapa Biosystems library preparation kit. Two hundred nanograms of sample DNA was sheared to $600 \mathrm{bp}$ using a Covaris LE220 focused-ultrasonicator. The sheared DNA fragments were size selected by double-SPRI and then the selected fragments were endrepaired, A-tailed, and ligated with Illumina-compatible sequencing adaptors from IDT containing a unique molecular index barcode for each sample library.

All the libraries were quantified using KAPA Biosystem's next-generation sequencing library qPCR kit and a Roche LightCycler 480 real-time PCR instrument. The quantified libraries were then multiplexed with other libraries, and the pool of libraries was prepared for sequencing on Illumina HiSeq sequencing platform utilizing a TruSeq paired-end cluster kit, v4, and Illumina's cBot instrument to generate a clustered flow cell for sequencing. Sequencing of the flow cell was performed on the Illumina
HiSeq 2500 sequencer using HiSeq TruSeq SBS sequencing kits, v4, following a $2 \times 150$ indexed run recipe.

\section{RNA-DNA co-extraction and sequencing}

Total RNA was extracted from a subset of 15 samples using the RNA PowerSoil Total RNA isolation kit (Qiagen). Soil samples (8 g) preserved in LifeGuard solution (Qiagen) were thawed on ice and centrifuged at $2500 \times g$ for $5 \mathrm{~min}$ to collect the soil at the bottom of the tubes. As a supernatant, the LifeGuard solution was extracted from the tubes and aliquoted into three $15-\mathrm{mL}$ conical tubes that were used to transfer three separate 2-g subsamples for later use. The remaining $2 \mathrm{~g}$ were split in half into two of the kit's bead tubes with pre-aliquoted bead solution (to disperse the cells and soil particles). The lysis solution (SR1) and the non-DNA organic and inorganic precipitation solution (SR2) were not added to the bead tube until all the subsamples to be processed in a given day had been aliquoted. Subsamples were kept at $-20{ }^{\circ} \mathrm{C}$ before transferring them to a $-80{ }^{\circ} \mathrm{C}$ freezer for permanent storage. The remainder of the extraction was carried out following the manufacturer's instructions. An RNA PowerSoil DNA elution accessory kit was used to co-extract DNA from the RNA capture columns, which was quantified as previously described. A DNase treatment was performed in all the RNA extracts with a TURBO DNA-free kit (Ambion) using $4 \mathrm{U}$ of TURBO DNase at $37^{\circ} \mathrm{C}$ for $30 \mathrm{~min}$. The absence of DNA was tested by PCR with universal primers to the SSU rRNA gene, and the integrity of the RNA was checked using a Bioanalyzer RNA 6000 Nano kit following the manufacturer's instructions. Total RNA was quantified before and after DNase treatments using a Qubit highsensitivity RNA assay (ThermoFisher Scientific). One of the RNA extracts (ERMLT590) did not yield enough RNA for sequencing.

Total RNA and DNA co-extracts were submitted for sequencing at the Joint Genome Institute in Walnut Creek, CA, where samples were subjected to a quality control check. rRNA was removed from $1 \mu \mathrm{g}$ of total RNA using Ribo-Zero ${ }^{\mathrm{TM}}$ rRNA Removal Kit (Illumina). Stranded cDNA libraries were generated using the Illumina Truseq Stranded mRNA Library Prep kit. The rRNA-depleted RNA was fragmented and reverse transcribed using random hexamers and SSII (Invitrogen) followed by second-strand synthesis. The fragmented cDNA was treated with end pair, A-tailing, adapter ligation, and 8 cycles of PCR. For low-input extracts, rRNA was removed from $100 \mathrm{ng}$ of total RNA using Ribo-Zero ${ }^{\text {TM }}$ rRNA Removal Kit (Illumina). Stranded cDNA libraries were generated using the Illumina Truseq Stranded mRNA Library Prep kit. The rRNAdepleted RNA was fragmented and reverse transcribed using random hexamers and SSII (Invitrogen) followed 
by second-strand synthesis. The fragmented cDNA was treated with end pair, A-tailing, adapter ligation, and 10 cycles of PCR. The prepared libraries were quantified using KAPA Biosystem's next-generation sequencing library qPCR kit and run on a Roche LightCycler 480 real-time PCR instrument. The quantified libraries were then multiplexed with other libraries, and the pool of libraries was prepared for sequencing on the Illumina HiSeq sequencing platform utilizing a TruSeq pairedend cluster kit, v4, and Illumina's cBot instrument to generate a clustered flow cell for sequencing. Sequencing of the flow cell was performed on the Illumina HiSeq 2500 sequencer using HiSeq TruSeq SBS sequencing kits, v4, following a $2 \times 150$ indexed run recipe.

\section{Metagenomes assembly and annotation and ribosomal protein L6 analysis}

Methods used for 2015 and 2016 metagenome assembly and annotation are described elsewhere [29]. In brief, after quality filtering, reads from individual samples were assembled separately using IDBA-UD v1.1.1 [30] with a minimum k-mer size of 40, a maximum k-mer size of 140 , and step size of 20 . Only contigs $>1 \mathrm{~Kb}$ were kept for further analyses. Gene prediction was done with Prodigal v2.6.3 [31] in meta mode, annotations obtained using USEARCH [32] against Uniprot [33], Uniref90 and KEGG [34], and 16S rRNA and tRNAs predicted as described in Diamond et al. [5]. Reads were mapped to the assemblies using Bowtie2 [35] and default settings to estimate coverage. To estimate the number of genomes potentially present across all 94 metagenomes, we used the ribosomal protein L6 as marker gene and RPxSuite (https://github.com/alexcritschristoph/RPxSuite) as described in Olm et al. [6]. L6 OTU clusters were considered "binned" if any L6 containing scaffold within an L6 OTU cluster across all samples was associated with a binned genome. L6 clusters were taxonomically classified using GraftM (https://doi.org/10.1093/nar/gky174) against all L6 sequences from the GTDB database (Release 05-RS95) with default parameters. Rank abundance curve plotting was accomplished using the ggplot2 [36] package in $\mathrm{R}$ [37].

\section{Genome binning, curation, and dereplication}

Annotated metagenomes from both years were uploaded onto ggKbase (https://ggkbase.berkeley.edu), where binning tools based on GC content, coverage, and winning taxonomy [38] were used for genome binning. These bins and additional bins that were obtained with the automated binners ABAWACA1 (https://github.com/ CK7/abawaca), ABAWACA2, MetaBAT [39], Maxbin2 [40], and Concoct [41] were pooled, and DAStool [42] was used for selection of the best set of bins from each sample as described by Diamond et al. [5]. Notably, no bins were recovered from sample ERMZT266 by any method.

Genomic bins were filtered based on completeness $\geq$ $70 \%$ of a set of 51 bacterial single copy genes (BSCG) if affiliated with Bacteria and a set of 38 archaeal singlecopy genes (ASCG); and a level of contamination $\leq 10 \%$ based on the corresponding list of single-copy genes [42]. Additionally, bins that were 59-68\% complete with a highest taxonomic level defined as Bacteria in ggKbase, or potential members of the candidate phyla radiation (CPR) were kept for further scrutiny. To obtain a set of genomes for visual curation in ggKbase, genomes were dereplicated at $99 \%$ ANI across samples located within a given floodplain using dRep [43] with the --ignoreGenomeQuality flag [43]. Any assembly error in the dereplicated set was addressed using ra2.py [44], and contigs that fell below the $1-\mathrm{Kb}$ length minimum after this step were removed from the bins. At this point, the level of completeness of CPR genomes was confirmed based on a list of 43 BSCG [7]. Genomes that did not meet the completeness thresholds post-assembly error correction and that were not affiliated with CPR or novel bacteria were removed from the analysis. Considering that bins changed as a result of this process, genes were re-predicted using Prodigal [31] in single mode, reads were mapped to the bins using Bowtie2 [45], and bins were reimported onto ggKbase. Visual inspection of taxonomic profile, GC content and to a minor extent coverage, allowed us to further reduce contamination. The final set of 248 curated bins from 2015 was dereplicated at 98\% ANI this time across floodplains including the --genomeInfo flag to take into account completeness and contamination in the process of representative bin selection. Within this set, genomes $\geq$ 90\% complete were deemed near-complete (Table S2, Additional file 2). Eight relatively low-coverage genomes fell just below the completeness requirement due to fragmentation after curation to remove possible local assembly errors; these were retained as they represent important taxonomic diversity.

Similarly, genomes reconstructed from floodplain L samples collected in 2016 that passed the completeness $(\geq 70 \%)$ and contamination thresholds $(\leq 10 \%)$ were visually inspected and improved in ggKbase. Assembly errors were corrected with ra2.py [44], and contigs that fell below the $1-\mathrm{Kb}$ length were removed, as well as genomes that did not pass the thresholds for completeness after assembly error correction. Genes were re-predicted using Prodigal [31] in single mode, and the final set of curated genomes were imported onto ggKbase.

To determine whether the same species were present in two different years, we pooled the genome set from 2015 and the curated 2016 set and dereplicated using dRep [43] at 95\% ANI including the --genomeInfo flag to take into account completeness and contamination in 
the process of representative bin selection [43]. In this set of genomes, 13 were reconstructed from a deeper depth (Table S3, Additional file 4). However, only 3 genomes were unique and the other 10 clustered with genomes reconstructed from the $\sim 10-25-\mathrm{cm}$ depth, indicating overlap between the species found at the two depths. Therefore, we kept these genomes for further analyses.

\section{Genome metabolic annotation}

We carefully chose a set of ecologically relevant proteins that catalyze geochemical transformations related to aerobic respiration, metabolism of $\mathrm{C} 1$ compounds, hydrogen metabolism, nitrogen cycling, and sulfur cycling (Table S4, Additional file 6). Hidden Markov Models (HMMs) for the majority of these proteins were obtained from KOfam, the customized HMM database of KEGG Orthologs (KOs) [46]. Custom-made HMMs targeting nitrite oxidoreductase subunits $\mathrm{A}$ and $\mathrm{B}$ (NxrA and NxrB), periplasmic cytochrome $c$ nitrite reductase (NirS, cd1-NIR heme-containing), cytochrome $c$-dependent nitric oxide reductase (NorC; cNOR), hydrazine dehydrogenase (HzoA), hydrazine synthase (HzsA), dissimilatory sulfite reductase D (DsrD), sulfide:quinone reductase (Sqr), sulfur dioxygenase (Sdo), ribulose-bisphosphate carboxylase (RuBisCO) form I and form II, and alcohol dehydrogenases (Pqq-XoxF$\mathrm{MxaF}$ ) were obtained from Anantharaman et al. [7]. $\mathrm{NiFe}$ and $\mathrm{FeFe}$ hydrogenases were predicted using HMMs from Méheust et al. [47] and assigned to functional groups following Matheus Carnevali et al. [38] (see Phylogenetic analyses subsection below for tree construction methods; Data S3, Additional file 12 and Data S4, Additional file 13; Table S9, Additional file 14 and Table S10, Additional file 15). No real group 4 membrane-bound NiFe hydrogenases were identified among the East River representative genomes (data not shown). HMMER3 [48] was used to annotate the dereplicated sets of genomes following predefined score cutoffs [46]. A subset (10\%) of the hits to all of these HMMs were visually checked to determine whether the cutoffs were appropriate for this dataset as described in Lavy et al. [49] and Jaffe et al. [50]. Only in the case of formate dehydrogenase (FdhA (K05299 and K22516), FdoG/FdhF/FdwA (K00123)) was the cutoff lowered to include additional hits.

For a protein to be considered potentially encoded in the genome, the catalytic subunit and the majority of the accessory subunits had to be detected by the corresponding HMMs at the established cutoffs. The implication for these function definitions is that in some cases even if some subunits that make up an enzyme were detected, the enzyme could have been deemed absent because a key part was missing (Table S4, Additional file 6). Similarly, pathways that require the activity of multiple enzymes were only detectable if all of the enzymes were present. Only in cases like the WoodJungdahl pathway, we required the majority of the genes to be present, taking into consideration genome completeness. Furthermore, if multiple enzymes could catalyze a given reaction (e.g., use $\mathrm{O}_{2}$ as a terminal electron acceptor), the presence of genes encoding one such enzyme in a genome would be indicative that this capacity was present in the genome. Additionally, if different pathways lead to the same biogeochemical transformation (e.g., $\mathrm{CO}_{2}$-fixation), the presence of genes encoding one of those pathways (or key enzymes) was considered as sufficient to indicate its presence (Table S4, Additional file 6). In a limited number of cases, a given pathway may also involve enzymes that are part of central metabolism or that are part of multiple pathways, and in these cases, we chose to define presence based on the key catalyst instead of the whole pathway (e.g., RuBisCO in the Calvin Benson pathway).

Carbohydrate-active enzymes were predicted using the Carbohydrate-Active enZYmes Database (CAZy; http:// www.cazy.org/) [10] (version 1.0) and dbCAN2 [51] (e value cutoff 1e-20).

\section{Genome coverage and detection}

Reads were mapped to the dereplicated set of bins using Bowtie2 [35] and a mismatch threshold of 2\% dissimilarity. Calculate_coverage.py (https://github.com/ christophertbrown/bioscripts/tree/master/ctbBio) was used to estimate the average number of reads mapping to each genome and the proportion of the genome that was covered by reads (breadth). Genomes with a coverage of at least $0.01 \mathrm{X}$ were considered to be detected in a given sample. The Hellinger transformation was used to account for differences in sequencing depth among samples and determine final genome abundance. To illustrate genome detection across samples, we used the ggplot2 package [36]. Genomes were clustered by average linkage using the Hellinger-transformed abundance across samples (from read mapping), and the samples were clustered by Euclidean distance in $\mathrm{R}$ [37].

\section{Phylogenetic analyses}

Two phylogenetic trees were constructed with a set of 14 ribosomal proteins (L2, L3, L4, L5, L6, L14, L15, L18, L22, L24, S3, S8, S17, and S19). One tree included Betaproteobacteria genomes from this study at the subspecies level (98\% ANI) and 1540 reference Betaproteobacteria genomes from the NCBI (Figure S3, Additional file 3 and Data S1, Additional file 5). The other tree included the set of 215 genomes dereplicated at 95\% ANI and 2228 
reference genomes from the NCBI genome database (Data S2, Additional file 9). For each genome, the ribosomal proteins were collected along the scaffold with the highest number of ribosomal proteins. A maximum-likelihood tree was calculated based on the concatenation of the ribosomal proteins as follows: Homologous protein sequences were aligned using MAFFT (version 7.390) (--auto option) [52], and alignments refined to remove gapped regions using Trimal (version 1.4.22) (--gappyout option) [53]. Tree reconstruction was performed using IQ-TREE (version 1.6.12) (as implemented on the CIPRES web server [54], using ModelFinder [55] to select the best model of evolution (LG + I + G4), and with 1000 ultrafast bootstrap [56]. Taxonomic affiliations were determined based on the closest reference sequences relative to the query sequences on the tree and extended to other members of the ANI cluster. In many cases, the phylogeny was not clear upon first inspection of the tree and additional reference genomes were added if publicly available. Phylogenetic trees for proteins of interest were reconstructed using the same methods described above, except with different sets of reference sequences. East River homologs in the dimethyl sulfoxide reductase (DMSOR) superfamily such as the catalytic subunit of formate dehydrogenase (FdhA), nitrite oxidoreductase (NxrA), membrane-bound nitrate reductase (NarG; $\mathrm{H}^{+}$-translocating), and periplasmic nitrate reductase subunit A (NapA) were confirmed by phylogeny on a tree with reference sequences from Méheust et al. [47] (Table S11, Additional file 16 and Data S5, Additional file 17). To distinguish form I and form II CODHs and other subtypes among homologs to K03520, we used Diamond's et al. [5] dataset, which comprises reference sequences from Quiza et al. [18] (Table S12, Additional file 18 and Data S6, Additional file 19). Similarly, homologs identified using the Pqq-XoxF-MxaF HMM for alcohol dehydrogenases were placed on a phylogenetic tree with reference sequences from Diamond's et al. [5] dataset, comprising references from Keltjens et al. [57] and Taubert et al. [58]. In this tree, all East River homologs were clustered with methanol dehydrogenases (Table S13, Additional file 20 and Data S7, Additional file 21) instead of other types of alcohol dehydrogenases. To distinguish between dissimilatory (bi)sulfite reductase oxidative or reductive bacterial types, DsrA and DsrB homologs from individual genomes were concatenated to each other, aligned, and added to a phylogenetic tree with reference sequences from Muller et al. [59] (Table S14, Additional file 22 and Data S8, Additional file 23).

\section{Community diversity and composition}

Diversity indices for each sample were calculated from the Hellinger transformed abundance table for the genome set at subspecies level (98\% ANI) using the vegan package in $R$ [60]. Species numbers and Shannon diversity per sample were quantified using the specnumber and vegdist functions of vegan, respectively (Figure S4, Additional file 3 ). An analysis of variance, implemented in the aov function in R [37], was used to test for significant differences in mean species number and Shannon diversity in relationship to the floodplain samples originated from. No significant differences in group means were detected considering a $p$ value $<0.05$ as significant.

To investigate community composition at the phylum/ class level as determined by phylogenetic analysis, the Hellinger-transformed abundance table for the genome set at the subspecies level (98\% ANI) was converted to a presence/absence table. The number of samples where each genome was detected was counted, and the number of genomes affiliated to a given taxon was summed by sample and plotted in R [37] with ggplot2 [36].

\section{Identification of a core floodplain microbiome}

To identify organisms that were a "core" or "shared" set across all sampled sites, we operationally defined a core set as (1) organisms that were not statistically associated with any specific floodplain using indicator species analysis (ISA) and (2) that were detected (displayed $\geq 0.01 \mathrm{X}$ coverage) in at least 89 of the 94 total samples (the 90th percentile for this level of presence across all 248 genomes). Indicator species analysis was performed on the log transformed coverage values that were filtered to include only coverage values $\geq 0.01 \mathrm{X}$ using the indicspecies package [61] in $\mathrm{R}$ version 3.5.2 [37] with 9999 permutations. All $p$ values for associations of an organism genome with a floodplain or group of floodplains were then subsequently corrected using False Discovery Rate with FDR $\leq 0.05$ being considered a significant association. This resulted in 42 genomes that were not statistically associated with any floodplain by ISA and were also detected in $\geq 89$ samples (Table S5, Additional file $7)$. For visualization of organism abundance profiles in relationship to their membership in the core floodplain microbiome, ISA clusters, and relative to the coefficient of variation of their coverage, Hellinger normalized coverage data was projected onto a two-dimensional space using Uniform Manifold Approximation and Projection (UMAP) implemented in the uwot package in $\mathrm{R}$ [62] using the following parameters: $\operatorname{umap}($ data $=$ coverage_data, n_neighbors $=15, \mathrm{nn} \_$method $=$"fnn", spread $=5$, min_dist $=0.01, \mathrm{n} \_$components $=2$, metric $=$ "euclidian", n_epochs $=1000)$.

\section{Identification of enriched metabolic functions in core floodplain microbiome}

Overrepresentation of metabolic functions within the set of genomes comprising the core floodplain microbiome 
$(n=42)$ was assessed using hypergeometric testing. The probability of observing the number of genomes in the core floodplain microbiome carrying each of 33 functions, given the total number of genomes with that function across our full genomic dataset $(n=248)$, was calculated using the phyper function in R [37]. Probabilities calculated across all metabolic functions were corrected for multiple testing using false discovery rate with the p.adjust function in R [37] and with FDR $\leq 0.05$ being considered a significant enrichment of a function in the core microbiome.

\section{Analysis of correlations among environmental variables} Correlations between numeric soil biogeochemical variables across samples were calculated using Spearman rank correlation implemented in the rcorr function of the Hmisc package in R (https://github.com/harrelfe/Hmisc). Correlations between variables were then plotted as a correlogram and ordered using hierarchical clustering with Ward's method using the corrplot package in R [63].

\section{Fourth-corner analysis}

A rlq fourth-corner analysis was performed on genome abundances, environmental data, and genome metabolic annotations using the R package ade4 [64]. Specifically, the pre-Hellinger-transformed genome abundance table was used for a correspondence analysis, the selected environmental variables (see Soil Chemistry and GIS) were used for a Hill-Smith analysis, and the genome metabolic annotations were used for PCA. A randomization test (as described by ter Braak et al. [65] and Dray et al. [8] was used to test the global significance of the traitenvironment relationships. The fourth-corner statistic was then calculated on the same inputs as the rlq analysis with 50,000 permutations and $p$ value adjustments using the FDR global methods. The results of the rlq fourth-corner analysis were plotted using the ggplot2 package [36].

\section{Metatranscriptomic analyses}

To determine differentially transcribed genes, potential levels of activity by phylum or class, most transcribed $\mathrm{CAZy}$, and most transcribed genes among key geochemical transformations, metatranscriptomic reads were mapped using Bowtie2 [35] to a set of high-quality draft genomes dereplicated at $95 \%$ (see above). Metagenomic reads from the subset of floodplain $\mathrm{L}$ sites that were sampled both in 2015 and 2016 (Table S1, Additional file 1) were also mapped to confirm high transcription levels were not due to higher gene abundance in 2016 . Read pairs were then filtered by a minimum identity of $95 \%$ to the reference with MAPQ $\geq 2$, and total number of mapped read pairs was counted for each gene. Counts for metabolic genes were analyzed with DESeq2 [9] to determine differential expression in response to soil organic carbon, and $p$ values were adjusted to correct for multiple hypothesis testing (FDR $<0.05$ ).

\section{GIS}

All GIS operations and cartographic visualizations were performed in QGIS v2.12.1 except where otherwise stated. The base remote-sensed imagery used was obtained from USDA NAIP (USDA-FSA Aerial Photography Field Office publication date 20171220; $1 \mathrm{~m}$ ground pixel resolution). Digital terrain model (DTM) at a ground resolution of $0.5 \mathrm{~m} /$ pixel was derived by airborne LiDAR data acquired by Quantum Spatial in collaboration with Eagle Mapping Ltd [66] (doi:10.21952/WTR/1412542) in 2015. All maps were projected using EPSG:26913 NAD83/ UTM zone 13N. Meander and adjacent river polygons were manually delineated in QGIS. The distance from a sample point to the manually delineated river polygons was calculated using the NNJoin tool. To calculate the sample distances to meander toe, lines were manually drawn between all samples and the meander toe perpendicular to river flow and distances calculated using NNJoin (Figure S6, Additional file 3 a). Similarly, to calculate sample distances to the middle of the meander, a line perpendicular to the meander toe line was drawn across the middle of the meander (Figure S6, Additional file 3 ). Sample distances to this line were also calculated using NNJoin and samples on the downstream side of the line were converted to negative values to indicate upstream and downstream sides of the meander. TPI is computed from the DTM as the difference between the elevation of a center point and the average elevation measured in the neighboring area $(3$ by $3 \mathrm{~m}$ ) [66]. To display genome abundances as used in the rlq fourth-corner analysis, filtered abundance values were chi-square transformed in $\mathrm{R}$ using the decostand in the vegan package and exported to display in QGIS. Spatial kriging of inorganic carbon was performed in $\mathrm{R}$. The manually delineated meander polygons were converted to SpatialPixelsDataFrame using the sp package. A simple variogram model was fit to the natural logtransformed inorganic carbon values with a spatial cutoff of $60 \mathrm{~m}$. Kriging was then performed using the sample points, the meander SpatialPixelsDataFrame, and the fitted variogram model. The natural log-transformed inorganic carbon values were then back transformed and the kriged map exported for visualization in QGIS. 


\section{Supplementary Information}

The online version contains supplementary material available at https://doi. org/10.1186/s40168-020-00957-z.

Additional file 1: Table S1. Sample sequencing and assembly information, and NCBI accession numbers.

Additional file 2: Table S2. Representative genomes (of 248 subspecies clusters at $98 \% \mathrm{ANI}$ ) information. This set of genomes was reconstructed from soil samples collected in September 2015.

Additional file 3: Figure S1. Rank abundance curve of rpL6 scaffolds (centroids for sequences clustered at $97.5 \%$ nucleotide identity) and their taxonomic assignment. Black ticks represent clusters with scaffolds that were binned in genomes. Clusters with $<5$ sequences were combined in "others". The taxonomic affiliation presented here corresponds to that of the Genome Taxonomy Database (GTDB release 05-RS95), while the taxonomy of the genomes was assigned based on phylogenetic placement in a tree of 14 concatenated ribosomal proteins. There may be discrepancies in the taxonomic assignments between the single rpL6 genes and the genomes, arising from the use of a different method to generate this figure. For example, Gammaproteobacteria in GTDB (https://gtdb.ecogenomic.org/) include Betaproteobacteria, and Deltaproteobacteria in NCBI include Myxococcota and Desulfobacterota, which are separate clades in GTDB. Scaffolds from multiple CPR (Patescibacteria) were not classified by GTDB and have been grouped as "Unk" or unclassified in this figure. Figure S2. Percent of samples within each floodplain where a genome was detected at the sub-species level (98\% ANI). Presence or absence was determined based on Hellinger transformed abundance (average coverage $\geq 0.01$ ). (a) Detection regardless of where the genome was reconstructed from. (b) Detection according to floodplain of origin. Genomes were detected in a higher number of samples from a given floodplain if they were reconstructed from a sample within that floodplain. Figure S3. Concatenated ribosomal proteins IQ-TREE of Betaproteobacteria at the sub-species level (98\% ANI) and $\sim 1540$ reference genomes from the NCBI. East River Betaproteobacteria are shown in bold magenta font (from this study) and violet font (from [49]). Some environmental sequences related to East River Betaproteobacteria are highlighted in orange, and next to the accession number is the environment of origin. Clades at the family level follow GTDB taxonomy [68] for additional reference. Figure S4. Diversity indices calculated for a set of representative genomes at the sub-species level (98\% ANI). Figure S5. Environmental variables used in fourth corner analysis: solid face chemistry from soil samples collected in 2015 including total carbon (TC; \%), inorganic carbon (IC; \%), organic carbon (OC; \%), total nitrogen (TN; \%), total carbon to total nitrogen ratio (OC:N) and measures associated with sample site locations: distance to river, elevation, easting, northing, topographic position index (TPI), and distance to the inner bank edge (or toe distance) and distance to middle of the meander-bound floodplain. Figure S6. Diagram representing imaginary lines used to determine distance to the middle of the meander-bound floodplain and distance to the inner bank edge (toe distance) as alternative measures of samples position on the floodplains. Figure S7. Spearman's correlation among environmental variables (2015). Figure S8. Number of reads mapped to genes encoding geochemically relevant functions in the species-level set of 215 genomes. (a) Number of DNA reads mapped from 2015 metagenomes and from 2016 metagenomes. This plot shows a high degree of correspondence between the two different years. (b) Number of RNA and DNA reads mapped from 2016 metatranscriptomes and 2015 metagenomes obtained from the same sampling sites. (c) Number of RNA and DNA reads from 2016 metatranscriptomes and metagenomes from the same subset of sampling sites. These plots show that in some cases there are high numbers of RNA reads mapping to low abundance genes (represented by low number of mapped DNA reads). Most obvious examples on figures $b$ and $c: M e D H$ : methanol dehydrogenase. $n \times r B$ : nitrite oxidoreductase subunit B. narH: membrane-bound nitrate reductase $\left(\mathrm{H}^{+}\right.$- translocating). fdoG, fdh $F, f d w A$ : $\mathrm{NAD}^{+}$-dependent formate dehydrogenase major subunit. oor AB: 2oxoglutarate/2-oxoacid ferredoxin oxidoreductase subunits alpha and beta. coxL: aerobic carbon monoxide dehydrogenase large subunit. Figure S9. Spearman's correlation among environmental variables (2016).
Additional file 4: Table S3. Representative genomes (of 215 species clusters at $95 \%$ ANI) information. This set of genomes includes genomes reconstructed from samples collected in September 2015 and September 2016.

Additional file 5. Data S1: Concatenated ribosomal proteins phylogenetic tree of Betaproteobacteria among 248 representative genomes of sub-species level clusters at $98 \%$ ANI.

Additional file 6: Table S4. Presence/Absence of gene homologs identified among 248 representative genomes using selected HMMs. Includes rules used to define functions.

Additional file 7: Table S5. Results of the indicator species analysis. Additional file 8: Table S6. Presence/Absence of functions among 248 representative genomes.

Additional file 9. Data S2. Concatenated ribosomal proteins phylogenetic tree of 215 representative genomes of species level clusters at $95 \%$ ANI.

Additional file 10: Table S7. Solid phase chemistry for subset of samples collected in September 2016 with paired metatranscriptomes.

Additional file 11: Table S8. Carbohydrate active enzymes (CAZys) in the glycosyl hydrolases $(\mathrm{GH})$, carbohydrate esterases (CE), polysaccharide lyases (PL), and auxiliary activities (AA) classes detected among the 215 representative genomes (e- value cut-off 1e-20).

Additional file 12. Data S3. Phylogenetic tree of NiFe hydrogenases groups 1, 2 and 3 reference sequences and homologs identified among unique sequences from both genome sets.

Additional file 13. Data S4. Phylogenetic tree of FeFe hydrogenases groups A, B and C reference sequences and homologs identified among unique sequences from both genome sets.

Additional file 14: Table S9. Unique sequences from both genome sets confirmed to be homologous to NiFe hydrogenases groups 1, 2 or 3 based on a phylogenetic analysis.

Additional file 15: Table S10. Unique sequences from both genome sets confirmed to be homologous to FeFe hydrogenases groups A, B or $C$ based on a phylogenetic analysis. Sequences identified among the 248 representative genomes were used in analyses based on presence/ absence. Sequences identified among the 215 representative genomes were used in transcript analyses.

Additional file 16: Table S11. Unique sequences from both genome sets confirmed to be homologous to FdhA/FdoG/FdhF/FdwA, NxrA, NapA, and NarG based on a phylogenetic analysis.

Additional file 17. Data S5. Phylogenetic tree of DMSOR superfamily reference sequences and homologs identified among unique sequences from both genome sets.

Additional file 18: Table S12. Unique sequences from both genome sets confirmed to be homologous to CoxL based on a phylogenetic analysis. Subtype is indicated next to the sequences.

Additional file 19. Data S6. Phylogenetic tree of CoxL reference sequences and homologs identified among unique sequences from both genome sets.

Additional file 20: Table S13. Unique sequences from both genome sets confirmed to be homologous to methanol dehydrogenases.

Additional File 21. Data S7. Phylogenetic tree of PQQ-containing alcohol dehydrogenases reference sequences and homologs identified among unique sequences from both genome sets.

Additional File 22: Table S14. Unique sequences from both genome sets homologous to DsrAB. Subtype is indicated next to the sequences.

Additional file 23. Data S8: Phylogenetic tree of concatenated DsrAB reference sequences and homologs identified among unique sequences from both genome sets.

\section{Acknowledgements}

We are grateful to Chad Hobson, David McGrath, and Rosemary Carrol for their support in the field during sample collection; to Joel Rowland (Los Alamos National Lab) and Helen Malenda (USGS) for their useful insights, 
and to Kate Lane, Brian C. Thomas, and Rohan Sachdeva for their technical support. We thank the Rocky Mountain Biological Laboratory for the lab space at the field site.

\section{Authors' contributions}

PBMC, JFB, KHW, and SH conceived the study. PBMC, JFB, ADT, KHW, MN, and $\mathrm{DD}$ conceived the experimental design. PBMC, JFB, AL, AS, and SL performed metagenome assembly, genome binning, and genomic data processing. PBMC and AL designed biogeochemical function analysis and performed gene annotations; and PBMC performed metabolic analyses. PBMC, MRO, SD, and ADT performed genome coverage data analysis. SD, $A D T$, and PBMC performed statistical analyses of genomic data. ACC and PBMC performed all metatranscriptomics analysis. RM and PBMC performed CAZy analysis on genomic data and phylogenetic analyses. WD and KHW performed solid-phase chemistry analyses. ADT, NF, and HW performed GIS based analyses. PBMC and JFB wrote the manuscript with input from NB, MN, PN, KHW, SH, SD, ACC, AL, ADT, MRO, and AS. The author(s) read and approved the final manuscript.

\section{Funding}

This work was supported as part of the Watershed Function Scientific Focus Area funded by the US Department of Energy, Office of Science, Office of Biological and Environmental Research under Award Number DE-AC0205CH11231. Sequencing was conducted at the Joint Genome Institute (a DOE Office of Science User Facility) under a CSP award.

\section{Availability of data and materials}

The datasets generated during and/or analyzed during the current study are available in NCBI's SRA repository; all metagenomes and metatranscriptomes can be accessed using the umbrella accession number (PRJNA630765). Table S1 (Additional file 1) includes NCBI Bioproject accession numbers for individual metagenomes metatranscriptomes. Representative genomes at the subspecies level (98\% ANI set) and the species level (95\% ANI set), in addition to the environmental data (metadata) that support the findings of this study are available in ESS-DIVE with the identifier DOI: https://doi.org/10. $15485 / 1631979$ [67]. Computer code not already cited in the manuscript is available upon request at any time.

\section{Declarations}

Ethics approval and consent to participate

Not applicable

\section{Consent for publication}

Not applicable

\section{Competing interests}

The authors declare that they have no competing interests.

\section{Author details}

${ }^{1}$ Department of Earth and Planetary Science, University of California, Berkeley, USA. ${ }^{2}$ Department of Environmental Science, Policy, and Management, University of California, Berkeley, CA, USA. ${ }^{3}$ Department of Plant and Microbial Biology, University of California, Berkeley, CA, USA. ${ }^{4}$ Innovative Genomics Institute, Berkley, CA, USA. ${ }^{5}$ Current affiliation: Department of Microbiology and Immunology, Stanford University, Palo Alto, CA, USA. ${ }^{6}$ Earth and Environmental Sciences Area, Lawrence Berkeley National Laboratory, Berkeley, CA, USA. ${ }^{7}$ Chan Zuckerberg Biohub, San Francisco, CA USA.

Received: 24 June 2020 Accepted: 6 December 2020

Published online: 22 May 2021

\section{References}

1. Viviroli D, Dürr HH, Messerli B, Meybeck M, Weingartner R. Mountains of the world, water towers for humanity: typology, mapping, and global significance. Water Resour Res. 2007;43(7). https://doi.org/10.1029/2 006 wr005653.

2. Immerzeel WW, Lutz AF, Andrade M, Bahl A, Biemans H, Bolch T, et al. Importance and vulnerability of the world's water towers. Nature. 2020; 577(7790):364-9. https://doi.org/10.1038/s41586-019-1822-y.
3. Hubbard SS, Williams KH, Agarwal D, Banfield J, Beller H, Bouskill N, et al The East River, Colorado, watershed: a mountainous community testbed for improving predictive understanding of multiscale hydrologicalbiogeochemical dynamics. Vadose Zone J. 2018;17(1):180061. https://doi. org/10.2136/vzj2018.03.0061.

4. Levin SA. The problem of pattern and scale in ecology: the Robert $\mathrm{H}$. MacArthur Award lecture. Ecology. 1992;73(6):1943-67. https://doi.org/10.23 07/1941447.

5. Diamond S, Andeer PF, Li Z, Crits-Christoph A, Burstein D, Anantharaman K, et al. Mediterranean grassland soil $\mathrm{C}-\mathrm{N}$ compound turnover is dependent on rainfall and depth, and is mediated by genomically divergent microorganisms. Nat Microbiol. 2019, 4(8). https://doi.org/10.1038/s41564-01 9-0449-y.

6. Olm MR, Crits-Christoph A, Diamond S, Lavy A, Matheus Carnevali PB, Banfield JF. Consistent metagenome-derived metrics verify and delineate bacterial species boundaries. mSystems. 2020;5(1):e00731-19. https://doi. org/10.1128/mSystems.00731-19.

7. Anantharaman K, Brown CT, Hug LA, Sharon I, Castelle CJ, Probst AJ, et al. Thousands of microbial genomes shed light on interconnected biogeochemical processes in an aquifer system. Nat Commun. 2016;7(1): 13219. https://doi.org/10.1038/ncomms13219.

8. Dray S, Choler P, Dolédec S, Peres-Neto PR, Thuiller W, Pavoine S, et al. Combining the fourth-corner and the RLQ methods for assessing trait responses to environmental variation. Ecology. 2014;95(1):14-21. https://doi. org/10.1890/13-0196.1.

9. Love Ml, Huber W, Anders S. Moderated estimation of fold change and dispersion for RNA-seq data with DESeq2. Genome Biol. 2014;15(12):550. https://doi.org/10.1186/s13059-014-0550-8.

10. Lombard V, Golaconda Ramulu H, Drula E, Coutinho PM, Henrissat B. The carbohydrate-active enzymes database (CAZy) in 2013. Nucleic Acids Res. 2014;42(Database issue):D490-D5. https://doi.org/10.1093/na r/gkt1178.

11. Dwivedi D, Steefel Cl, Arora B, Newcomer M, Moulton JD, Dafflon B, et al. Geochemical exports to river from the intrameander hyporheic zone under transient hydrologic conditions: East River Mountainous Watershed, Colorado. Water Resour Res. 2018:54(10):8456-77. https://doi.org/10.1029/2 018WR023377.

12. Fox PM, Bill M, Heckman K, Conrad M, Anderson C, Keiluweit M, et al. Shale as a Source of organic carbon in floodplain sediments of a mountainous watershed. J Geophys Res. 2020;125(2):e2019JG005419. https://doi.org/10.1 029/2019jg005419.

13. Ander P, Eriksson K-E. Methanol formation during lignin degradation by Phanerochaete chrysosporium. Appl Microbiol Biotechnol. 1985:21(1):96-102. https://doi.org/10.1007/BF00252369.

14. Dušková D, Marounek M. Fermentation of pectin and glucose, and activity of pectin-degrading enzymes in the rumen bacterium Lachnospira multiparus. Lett Appl Microbiol. 2001;33(2):159-63. https://doi.org/10.1046/ j.1472-765x.2001.00970.x

15. Argiroff WA, Zak DR, Lanser CM, Wiley MJ. Microbial community functional potential and composition are shaped by hydrologic connectivity in riverine floodplain soils. Microb Ecol. 2017:73(3):630-44. https://doi.org/10.1007/ s00248-016-0883-9.

16. Wan J, Tokunaga TK, Williams KH, Dong W, Brown W, Henderson AN, et al. Predicting sedimentary bedrock subsurface weathering fronts and weathering rates. Scientific Reports. 2019;9(1):17198. https://doi.org/10.1038/ s41598-019-53205-2.

17. King GM, Weber CF. Distribution, diversity and ecology of aerobic COoxidizing bacteria. Nat Rev Microbiol. 2007;5(2):107-18. https://doi.org/10.1 038/nrmicro1595.

18. Quiza L, Lalonde I, Guertin C, Constant P. Land-use influences the distribution and activity of high affinity CO-oxidizing bacteria associated to type l-coxL genotype in soil. Front Microbiol. 2014;5(271). https://doi.org/1 0.3389/fmicb.2014.00271

19. Cordero PRF, Bayly K, Man Leung P, Huang C, Islam ZF, Schittenhelm RB, et al. Atmospheric carbon monoxide oxidation is a widespread mechanism supporting microbial survival. ISME J. 2019;13(11):2868-81. https://doi.org/1 0.1038/s41396-019-0479-8

20. Boutard M, Cerisy T, Nogue P-Y, Alberti A, Weissenbach J, Salanoubat M, et al. Functional diversity of carbohydrate-active enzymes enabling a bacterium to ferment plant biomass. PLoS Genet. 2014;10(11):e1004773. https://doi.org/10.1371/journal.pgen.1004773. 
21. Houlton BZ, Morford SL, Dahlgren RA. Convergent evidence for widespread rock nitrogen sources in Earth's surface environment. Science. 2018; 360(6384):58-62. https://doi.org/10.1126/science.aan4399.

22. Winnick MJ, Carroll RWH, Williams KH, Maxwell RM, Dong W, Maher K. Snowmelt controls on concentration-discharge relationships and the balance of oxidative and acid-base weathering fluxes in an alpine catchment, East River, Colorado. Water Resour Res. 2017;53(3):2507-23. https://doi.org/10.1002/2016WR019724.

23. Bernal S, Hedin LO, Likens GE, Gerber S, Buso DC. Complex response of the forest nitrogen cycle to climate change. Proc Natl Acad Sci U S A. 2012; 109(9):3406-11. https://doi.org/10.1073/pnas.1121448109.

24. Sebestyen SD, Boyer EW, Shanley JB, Kendall C, Doctor DH, Aiken GR, et al. Sources, transformations, and hydrological processes that control stream nitrate and dissolved organic matter concentrations during snowmelt in an upland forest. Water Resour Res. 2008:44, 44(12, 12). https://doi.org/10.102 9/2008wr006983.

25. Rütting T, Boeckx P, Müller C, Klemedtsson L. Assessment of the importance of dissimilatory nitrate reduction to ammonium for the terrestrial nitrogen cycle. Biogeosciences. 2011;8(7):1779-91. https://doi.org/10.5194/bg-8-1 779-2011.

26. Hefting M, Clément JC, Dowrick D, Cosandey AC, Bernal S, Cimpian C, et al. Water table elevation controls on soil nitrogen cycling in riparian wetlands along a European climatic gradient. Biogeochemistry. 2004;67(1):113-34. https://doi.org/10.1023/B:BIOG.0000015320.69868.33.

27. Pribulick CE, Foster LM, Bearup LA, Navarre-Sitchler AK, Williams KH, Carroll $\mathrm{RWH}$, et al. Contrasting the hydrologic response due to land cover and climate change in a mountain headwaters system. Ecohydrology. 2016;9(8): 1431-8. https://doi.org/10.1002/eco.1779.

28. Albertsen M, Hugenholtz P, Skarshewski A, Nielsen KL, Tyson GW, Nielsen $\mathrm{PH}$. Genome sequences of rare, uncultured bacteria obtained by differential coverage binning of multiple metagenomes. Nat Biotechnol. 2013;31(6): 533-8. https://doi.org/10.1038/nbt.2579.

29. Lavy A, McGrath DG, Matheus Carnevali PB, Wan J, Dong W, Tokunaga TK, et al. Microbial communities across a hillslope-riparian transect shaped by proximity to the stream, groundwater table, and weathered bedrock. Ecol Evol. 2019;0(0). https://doi.org/10.1002/ece3.5254.

30. Peng Y, Leung HCM, Yiu SM, Chin FYL. IDBA-UD: a de novo assembler for single-cell and metagenomic sequencing data with highly uneven depth. Bioinformatics. 2012;28(11):1420-8. https://doi.org/10.1093/ bioinformatics/bts174.

31. Hyatt D, Chen G-L, LoCascio PF, Land ML, Larimer FW, Hauser LJ. Prodigal: prokaryotic gene recognition and translation initiation site identification. BMC Bioinformatics. 2010;11(1):119. https://doi.org/10.1186/1471-2105-11-119.

32. Westcott SL, Schloss PD. De novo clustering methods outperform referencebased methods for assigning $16 \mathrm{~S}$ rRNA gene sequences to operational taxonomic units. PeerJ. 2015;3:e1487. https://doi.org/10.7717/peerj.1487.

33. Consortium TU. UniProt: a worldwide hub of protein knowledge. Nucleic Acids Res. 2018;47(D1):D506-D15. https://doi.org/10.1093/nar/gky1049.

34. Kanehisa M, Goto S. KEGG: Kyoto Encyclopedia of Genes and Genomes. Nucleic Acids Res. 2000;28(1):27-30. https://doi.org/10.1093/nar/28.1.27.

35. Langmead B, Salzberg SL. Fast gapped-read alignment with Bowtie 2. Nat Methods. 2012;9(4):357-9. https://doi.org/10.1038/nmeth.1923.

36. Wickham H. ggplot2: elegant graphics for data analysis: Springer; 2016

37. Core R. Development Team: R: a language and environment for statistical computing. In: R Foundation for Statistical Computing; 2015.

38. Matheus Carnevali PB, Schulz F, Castelle CJ, Kantor RS, Shih PM, Sharon I, et al. Hydrogen-based metabolism as an ancestral trait in lineages sibling to the Cyanobacteria. Nat Commun. 2019;10(1):463. https://doi.org/10.1038/ s41467-018-08246-y.

39. Kang DD, Froula J, Egan R, Wang Z. MetaBAT, an efficient tool for accurately reconstructing single genomes from complex microbial communities. Peer」. 2015;3:e1165. https://doi.org/10.7717/peerj.1165.

40. Wu Y-W, Simmons BA, Singer SW. MaxBin 2.0: an automated binning algorithm to recover genomes from multiple metagenomic datasets. Bioinformatics. 2016;32(4):605-7. https://doi.org/10.1093/bioinformatics/ btv638.

41. Alneberg J, Bjarnason BS, de Bruijn I, Schirmer M, Quick J, ljaz UZ, et al. Binning metagenomic contigs by coverage and composition. Nat Methods. 2014;11(11):1144-6. https://doi.org/10.1038/nmeth.3103.

42. Sieber CMK, Probst AJ, Sharrar A, Thomas BC, Hess M, Tringe SG, et al. Recovery of genomes from metagenomes via a dereplication, aggregation and scoring strategy. Nat Microbiol. 2018;3(7):836-43. https://doi.org/10.103 8/s41564-018-0171-1.

43. Olm MR, Brown CT, Brooks B, Banfield JF. dRep: a tool for fast and accurate genomic comparisons that enables improved genome recovery from metagenomes through de-replication. ISME J. 2017;11(12):2864-8. https:// doi.org/10.1038/ismej.2017.126.

44. Brown CT, Hug LA, Thomas BC, Sharon I, Castelle CJ, Singh A, et al. Unusual biology across a group comprising more than 15\% of domain Bacteria. Nature. 2015;523(7559):208-11. https://doi.org/10.1038/nature14486.

45. Keel BN, Snelling WM. Comparison of Burrows-Wheeler transform-based mapping algorithms used in high-throughput whole-genome sequencing: application to Illumina data for livestock genomes. Front Genet. 2018;9:35. https://doi.org/10.3389/fgene.2018.00035.

46. Aramaki T, Blanc-Mathieu R, Endo H, Ohkubo K, Kanehisa M, Goto S, et al. KofamKOALA: KEGG ortholog assignment based on profile HMM and adaptive score threshold. bioRxiv. 2019:602110. https://doi.org/10.1101/ 602110

47. Méheust R, Castelle CJ, Carnevali PBM, Farag IF, He C, Chen L-X, et al. Groundwater Elusimicrobia are metabolically diverse compared to gut microbiome Elusimicrobia and some have novel nitrogenase-like gene clusters. ISME J. 2020;14(12):2907-22. https://doi.org/10.1038/s41396-020-071 6-1.

48. Pagnuco IA, Revuelta MV, Bondino HG, Brun M, ten Have A. HMMER Cut-off Threshold Tool (HMMERCTTER): supervised classification of superfamily protein sequences with a reliable cut-off threshold. PloS One. 2018;13(3): e0193757. https://doi.org/10.1371/journal.pone.0193757.

49. Lavy A, Matheus Carnevali PB, Keren R, Bill M, Wan J, Tokunaga TK, et al. Taxonomically and metabolically distinct microbial communities with depth and across a hillslope to riparian zone transect. bioRxiv. 2019:768572. https://doi.org/10.1101/768572

50. Jaffe AL, Castelle CJ, Matheus Carnevali PB, Gribaldo S, Banfield JF. The rise of diversity in metabolic platforms across the candidate phyla radiation. BMC Biol. 2020;18(1):69. https://doi.org/10.1186/s12915-020-00804-5.

51. Zhang $H$, Yohe $T$, Huang $L$, Entwistle $S$, Wu P, Yang Z, et al. dbCAN2: a meta server for automated carbohydrate-active enzyme annotation. Nucleic Acids Res. 2018;46(W1):W95-W101. https://doi.org/10.1093/nar/gky418.

52. Katoh K, Standley DM. A simple method to control over-alignment in the MAFFT multiple sequence alignment program. Bioinformatics. 2016;32(13): 1933-42. https://doi.org/10.1093/bioinformatics/btw108.

53. Capella-Gutiérrez S, Silla-Martínez JM, Gabaldón T. trimAl: a tool for automated alignment trimming in large-scale phylogenetic analyses. Bioinformatics. 2009; 25(15):1972-3. https://doi.org/10.1093/bioinformatics/btp348.

54. Miller MA, Pfeiffer W, Schwartz T. Creating the CIPRES Science Gateway for inference of large phylogenetic trees. In: Proceedings of the Gateway Computing Environments Workshop (GCE). New Orleans; 2010. p. 1-8. https://www.phylo.org/index.php/.

55. Kalyaanamoorthy S, Minh BQ, Wong TK, von Haeseler A, Jermiin LS. ModelFinder: fast model selection for accurate phylogenetic estimates. Nat Methods. 2017;14(6):587-9. https://doi.org/10.1038/nmeth.4285.

56. Hoang DT, Chernomor O, Von Haeseler A, Minh BQ, Vinh LS. UFBoot2: improving the ultrafast bootstrap approximation. Mol Biol Evol. 2018;35(2): 518-22. https://doi.org/10.1093/molbev/msx281.

57. Keltjens JT, Pol A, Reimann J. Op den Camp HJM. PQQ-dependent methanol dehydrogenases: rare-earth elements make a difference. Appl Microbiol Biotechnol. 2014;98(14):6163-83. https://doi.org/10.1007/s00253014-5766-8.

58. Taubert M, Grob C, Howat AM, Burns OJ, Dixon JL, Chen Y, et al. XoxF encoding an alternative methanol dehydrogenase is widespread in coastal marine environments. Environ Microbiol. 2015;17(10):3937-48. https://doi. org/10.1111/1462-2920.12896.

59. Müller AL, Kjeldsen KU, Rattei T, Pester M, Loy A. Phylogenetic and environmental diversity of DsrAB-type dissimilatory (bi)sulfite reductases. ISME J. 2014;9(5):1152-65. https://doi.org/10.1038/ismej.2014.208.

60. Oksanen J, Blanchet F, Kindt R, Legendre P, Minchin P, O'Hara R, et al. Vegan: community ecology package: $R$ package version 2.2-0; 2014. p. 10.

61. Cáceres MD, Legendre P. Associations between species and groups of sites: indices and statistical inference. Ecology. 2009;90(12):3566-74. https://doi. org/10.1890/08-1823.1.

62. Melville J. uwot: The uniform manifold approximation and projection (UMAP) method for dimensionality reduction. $R$ package version 00 09010; 2019. 
63. Wei T, Simko V. R package" corrplot": visualization of a correlation matrix (Version 0.84). Retrived from, https://github.com/taiyun/corrplot. 2017.

64. Dray S, Dufour A-B. The ade4 package: implementing the duality diagram for ecologists. J Stat Softw. 2007;22(4):1-20.

65. Ter Braak CJ, Cormont A, Dray S. Improved testing of species traitsenvironment relationships in the fourth-corner problem. Ecology. 2012;93(7): 1525-6. https://doi.org/10.1890/12-0126.1.

66. Falco N, Wainwright $\mathrm{H}$, Dafflon B, Léger E, Peterson J, Steltzer $\mathrm{H}$, et al. Investigating microtopographic and soil controls on a mountainous meadow plant community using high-resolution remote sensing and surface geophysical data. J Geophys Res. 2019;124(6):1618-36. https://doi. org/10.1029/2018jg004394

67. Matheus Carnevali P, Hobson C, Geller-McGrath D, Dong W, Falco N, Wainwright $\mathrm{H}$, et al. Genome-resolved metagenomics and metatranscriptomics of microbial communities in three meander-bound floodplain soils along the East River, Colorado. In: Environmental system science data infrastructure for a virtual ecosystem; 2020.

68. Parks DH, Chuvochina M, Chaumeil PA, Rinke C, Mussig AJ, Hugenholtz P. A complete domain-to-species taxonomy for Bacteria and Archaea. Nat Biotechnol. 2020;38(9):1079-86. https://doi.org/10.1038/s41587-020-0501-8.

\section{Publisher's Note}

Springer Nature remains neutral with regard to jurisdictional claims in published maps and institutional affiliations.

Ready to submit your research? Choose BMC and benefit from:

- fast, convenient online submission

- thorough peer review by experienced researchers in your field

- rapid publication on acceptance

- support for research data, including large and complex data types

- gold Open Access which fosters wider collaboration and increased citations

- maximum visibility for your research: over $100 \mathrm{M}$ website views per year

At BMC, research is always in progress.

Learn more biomedcentral.com/submissions 